\title{
The Herschel Reference Survey
}

\author{
A. Boselli,${ }^{1}$ S. Eales, ${ }^{2}$ L. Cortese,${ }^{2}$ G. Bendo,${ }^{3}$ P. Chanial,${ }^{3}$ V. Buat,${ }^{1}$ J. Davies,,${ }^{2}$ R. Auld, ${ }^{2}$ E. Rigby,,${ }^{4}$ M. Baes,${ }^{5}$ \\ M. Barlow, ${ }^{6}$ J. Bock,,${ }^{7}$ M. Bradford,${ }^{7}$ N. Castro-Rodriguez,${ }^{8}$ S. Charlot,${ }^{9}$ D. Cllements, ${ }^{3}$ D. Cormier, ${ }^{10}$ \\ E. Dwek, ${ }^{11}$ D. Elbaz,${ }^{10}$ M. Galametz,${ }^{10}$ F. Galliano, ${ }^{12}$ W. Gear,${ }^{2}$ J. Glenn,${ }^{13}$ H. Gomez, ${ }^{2}$ M. Griffin, ${ }^{2}$ \\ S. Hony, ${ }^{10}$ K. Isaak, ${ }^{2}$ L. Levenson,${ }^{7}$ N. Lu,,${ }^{7}$ S. Madden,,${ }^{10}$ B. O'Halloran,${ }^{3}$ K. OKamura,${ }^{10}$ \\ S. Oliver, ${ }^{14}$ M. Page,${ }^{15}$ P. Panuzzo, ${ }^{10}$ A. Papageorgiou, ${ }^{2}$ T. Parkin, ${ }^{16}$ I. Perez-Fournon,${ }^{8}$ \\ M. Pohlen, ${ }^{2}$ N. Rangwala,,${ }^{13}$ H. Roussel, ${ }^{9}$ A. Rykala, ${ }^{2}$ N. Sacchi,${ }^{17}$ M. Sauvage,,${ }^{10}$ \\ B. Schulz, ${ }^{18}$ M. Schirm, ${ }^{16}$ M. W. L. Smith, ${ }^{2}$ L. SPinoglio, ${ }^{17}$ J. Stevens,,${ }^{19}$ \\ M. SyMEONIDIS, ${ }^{19}$ M. VACCARI, ${ }^{20}$ L. VigrouX,${ }^{9}$ C. WILSON,${ }^{16}$ \\ H. WozNIAK, ${ }^{21}$ G. Wright, ${ }^{19}$ AND W. ZeILINGER ${ }^{22}$ \\ Received 2008 August 17; accepted 2010 January 26; published 2010 February 26
}

\begin{abstract}
The Herschel Reference Survey is a Herschel guaranteed time key project and will be a benchmark study of dust in the nearby universe. The survey will complement a number of other Herschel key projects including large cosmological surveys that trace dust in the distant universe. We will use Herschel to produce images of a statistically-complete sample of 323 galaxies at 250,350 , and $500 \mu \mathrm{m}$. The sample is volume-limited, containing sources with distances between 15 and $25 \mathrm{Mpc}$ and flux limits in the $K$ band to minimize the selection effects associated with dust and with young high-mass stars and to introduce a selection in stellar mass. The sample spans the whole range of morphological types (ellipticals to late-type spirals) and environments (from the field to the center of the Virgo Cluster) and as such will be useful for other purposes than our own. We plan to use the survey to investigate (i) the dust content of galaxies as a function of Hubble type, stellar mass, and environment; (ii) the connection between the dust content and composition and the other phases of the interstellar medium; and (iii) the origin and evolution of dust in galaxies. In this article, we describe the goals of the survey, the details of the sample and some of the auxiliary observing programs that we have started to collect complementary data. We also use the available multifrequency data to carry out an analysis of the statistical properties of the sample.
\end{abstract}

Online material: color figures

\section{INTRODUCTION}

Understanding the processes that govern the formation and evolution of galaxies is one of the major challenges of modern

\footnotetext{
${ }^{1}$ Laboratoire d'Astrophysique de Marseille, UMR6110 CNRS, 38 rue F. Joliot-Curie, F-13388 Marseille, France.

${ }^{2}$ School of Physics and Astronomy, Cardiff University, Queens Buildings The Parade, Cardiff CF24 3AA, UK.

${ }^{3}$ Astrophysics Group, Imperial College, Blackett Laboratory, Prince Consort Road, London SW7 2AZ, UK.

${ }^{4}$ School of Physics \& Astronomy, University of Nottingham, University Park, Nottingham NG7 2RD, UK.

${ }^{5}$ Sterrenkundig Observatorium, Universiteit Gent, Krijgslaan 281 S9, B-9000 Gent, Belgium.

${ }^{6}$ Department of Physics and Astronomy, University College London, Gower Street, London WC1E 6BT, UK.

${ }^{7}$ Jet Propulsion Laboratory, Pasadena, CA 91109, Department of Astronomy, California Institute of Technology, Pasadena, CA 91125.

${ }^{8}$ Instituto de Astrofísica de Canarias, C/Va Lctea s/n, E-38200 La Laguna, Spain.

${ }^{9}$ Institut d'Astrophysique de Paris, UMR7095 CNRS, Université Pierre \& Marie Curie, 98 bis Boulevard Arago, F-75014 Paris, France.
}

astronomy. Ideally this work can be done by analyzing and comparing the physical, structural and kinematical properties of different objects at various epochs to model predictions. How primordial galaxies transform their huge gas reservoir into stars

\footnotetext{
${ }^{10}$ Laboratoire AIM, CEA/DSM-CNRS-Université Paris Diderot, Irfu/Service d'Astrophysique, 91191 Gif sur Yvette, France.

${ }^{11}$ Observational Cosmology Lab, NASA Goddard Space Flight Center, Greenbelt, MD 20771.

${ }^{12}$ Department of Astronomy, University of Maryland, College Park, MD 20742.

${ }^{13}$ Department of Astrophysical and Planetary Sciences, University of Colorado, Boulder, CO 80309.

${ }^{14}$ Astronomy Centre, Department of Physics and Astronomy, University of Sussex, UK.

${ }^{15}$ Mullard Space Science Laboratory, University College London, Holmbury St Mary, Dorking, Surrey RH5 6NT, UK.

${ }^{16}$ Dept. of Physics \& Astronomy, McMaster University, Hamilton, Ontario L8S 4M1, Canada.

${ }^{17}$ Istituto di Fisica dello Spazio Interplanetario, INAF, Via del Fosso del Cavaliere 100, I-00133 Rome, Italy.

${ }^{18}$ Infrared Processing and Analysis Center, California Institute of Technology, Pasadena, CA 91125.
} 
and become the objects that we now observe in the local universe is a key question. A wide coverage of the electromagnetic spectrum to probe the atomic (21 cm line) and molecular (generally through the $2.6 \mathrm{~mm} \mathrm{CO}$ line) gas components, the different stellar populations (UV to near-IR spectral range), the dust (mid- and far-IR) and the electrons in magnetic fields (radio continuum) is necessary to address this important question.

The importance of dust resides in the fact that it is formed from the aggregation of the metals produced in the latest phases of stellar evolution, and contains approximately half the metals in the interstellar medium (Whittet 1992). Injected into the interstellar medium (ISM) by stellar winds and supernovae explosions, dust acts as a catalyst in the process of transformation of the atomic to molecular hydrogen necessary to feed star formation (Hollenbach \& Salpeter 1971; Duley \& Williams 1986). Dust also contributes to the shielding of the UV radiation field, preventing the dissociation of molecular clouds, and thus playing a major role in the energetic equilibrium of the ISM (Hollenbach \& Tielens 1997). Dust contributes to the cooling and heating of the ISM in photodissociation regions through photoelectric heating. Furthermore, by absorbing the stellar light, dust modifies our view of the different stellar components (e.g., Buat \& Xu 1996): because dust obscuration is so important in star-forming regions, the emission from dust is one of the most powerful tracers of the star formation activity in galaxies (Kennicutt 1998; Hirashita et al. 2003). Dust emission can therefore be used to study the relationship between the gas surface density and the star formation activity, generally known as the Schmidt law.

The importance of dust in the study of the formation and evolution of galaxies became evident after the IRAS space mission which provided us with a whole sky coverage in four infrared bands. Despite its low sensitivity and poor angular resolution, IRAS detected tens thousands of extragalactic sources (Soifer et al. 1987). The IRAS survey showed that the stellar light in some galaxies is so heavily obscured that the only way to determine their star formation activity is through the dust emission itself. Among these highly obscured galaxies, the Ultraluminous Infrared Galaxies (ULIRGs) are the most actively star-forming objects in the local universe. With their improved sensitivity, spectral and angular resolution, other space missions such as ISO and Spitzer have brought a better understanding of the chemical and physical properties of interstellar dust in a wide range of different galactic and extragalactic sources. IRAS, ISO, Spitzer, and the recently launched AKARI satellite, however, are sensi-

\footnotetext{
${ }^{19}$ Centre for Astrophysics Research, Science and Technology Research Centre, University of Hertfordshire, College Lane, Herts AL10 9AB, UK.

${ }^{20}$ University of Padova, Department of Astronomy, Vicolo Osservatorio 3, I-35122 Padova, Italy.

${ }^{21}$ Observatoire Astronomique de Strasbourg, UMR 7550 Université de Strasbourg-CNRS, 11, rue de l'Université, F-67000 Strasbourg, France.

${ }^{22}$ Institut für Astronomie, Universität Wien, Türkenschanzstr. 17, A-1180 Wien, Austria.
}

tive to dust emitting in the mid- $(\sim 5 \mu \mathrm{m})$ to far- $(\leq 200 \mu \mathrm{m})$ infrared domain. The 5-70 $\mu \mathrm{m}$ spectral range corresponds to the emission of the hot dust generally associated to star formation, while at longer wavelengths, up to $1 \mathrm{~mm}$, the contribution of cold dust becomes more and more important (Draine et al. 2007). The emission of the ISM in the range $3 \mu \mathrm{m} \lesssim \lambda \lesssim 15 \mu \mathrm{m}$ is generally dominated by polycyclic aromatic hydrocarbons (PAHs). Between $10 \mu \mathrm{m}$ and $\lesssim 70 \mu \mathrm{m}$ the dust emission is usually due to very small grains, while that at longer wavelengths $(70 \mu \mathrm{m} \lesssim \lambda \lesssim$ $1000 \mu \mathrm{m}$ ) is probably produced by large grains of graphite and silicate in thermal equilibrium with the UV and optical photons of the interstellar radiation field (Désert et al. 1990; Dwek et al. 1997; Zubko et al. 2004; Draine \& Li 2007).

There is, however, strong empirical evidence to suggest that much of the dust in normal galaxies has been missed by previous space missions because it is too cold to radiate in the mid- and far-infrared. Devereux \& Young (1990), for example, showed that when the dust masses of galaxies were estimated from IRAS data, the gas-to-dust ratios were $\simeq 10$ times higher than the Galactic value, implying that $90 \%$ of the dust in galaxies is too cold to radiate significantly in the IRAS bands. ISO $200 \mu \mathrm{m}$ images of nearby galaxies revealed the presence of cold $(\sim 20 \mathrm{~K})$ dust in the external parts of galaxies (Alton et al. 1998). Even Spitzer, with its longer wavelength coverage, is only sensitive to dust that is warmer than $\simeq 15 \mathrm{~K}$ (Bendo et al. 2003; Draine \& Li 2007; Draine et al. 2007). Simple estimates show that the temperature of a dust grain in thermal equilibrium in the average interstellar radiation field is $\sim 20 \mathrm{~K}$, which would produce a modified black body spectrum with a peak at $\sim 200 \mu \mathrm{m}$ with a flux rapidly decreasing at longer wavelengths (Boselli et al. 2003a; Dale et al. 2005, 2007; Draine \& Li 2007; Draine et al. 2007). It is important to remember, however, that most of the dust in a galaxy is likely to be at a much lower temperature than $20 \mathrm{~K}$ because it is self-shielded, and so unaffected from the interstellar radiation field. For example, in molecular clouds the temperature of the dust is usually well below the canonical $20 \mathrm{~K}$ value once the average dust extinction $\left(A_{V}\right)$ is greater than one, unless it is close to a newly formed star (Ward-Thompson et al. 2002).

The submillimeter waveband (200-1000 $\mu \mathrm{m})$ is crucial for detecting this missing cold dust component and thus for making accurate estimates of the total dust mass. Mass estimates made from far-infrared measurements are highly uncertain because of the strong dependence of flux on dust temperature which is difficult to determine using data on the Wien side of the black body peak. At long wavelengths (Rayleigh-Jeans domain) the emissivity depends only on the first power of the dust temperature, making it possible to use a submillimeter flux to make an accurate estimate of the mass of dust (Eales et al. 1989; Galliano et al. 2003; 2005).

Submillimeter continuum observations of galaxies have been made previously, in particular with the ground-based submillimeter cameras, such as SCUBA on the James Clerk Maxwell 
Telescope. These have confirmed that galaxies do indeed contain a large amount of cold dust (Dunne \& Eales 2001; Galliano et al. 2003). Until now, the largest submillimeter survey has been the SCUBA Local Universe and Galaxy Survey (SLUGS), a survey of $\simeq 200$ galaxies in two samples, one selected from the IRAS survey and one selected at optical wavelengths. The survey produced the first estimates of the submillimeter luminosity and dust-mass (the space density of galaxies as a function of dust mass) functions (Dunne et al. 2000; Vlahakis et al. 2005), and also indicated that some ellipticals are surprisingly strong submillimeter sources. Its limitation, and the limitation of all ground-based submillimeter observations, is that of sensitivity: SLUGS struggled to detect galaxies not already detected by IRAS.

The solution is to go into space and the Herschel space telescope is finally making this possible. In particular the relatively large field of view, high sensitivity, and coverage of a waveband $(250-500 \mu \mathrm{m})$ in which galaxies are intrinsically bright, make SPIRE on Herschel the ideal instrument for a study of all extragalactic sources (Griffin et al. 2006, 2007; Wasket et al. 2007). SPIRE has the sensitivity to map large areas of the sky down to the confusion limit in quite modest integration times, providing submillimeter data for large samples of galaxies at different redshifts, and is thus well adapted for pointed observations for all kind of nearby extragalactic sources.

The SPIRE consortium has defined a number of coordinated guaranteed time programs to get the best possible benefit of the unique Herschel facilities for the study of galaxy evolution. These include deep cosmological surveys, pointed observations of local galaxies and surveys of representative regions in the Milky Way. To better characterize the dust properties in the local universe, the SPIRE extragalactic group has selected a volumelimited sample of 323 galaxies to be observed in guaranteed time in the three SPIRE bands at 250, 350, and $500 \mu \mathrm{m}$. The importance of the local universe resides in the fact that it represents the endpoint of galaxy evolution, providing important boundary conditions to models and simulations. Furthermore, at $\leq 30 \mathrm{Mpc}$ the angular resolution of SPIRE (a couple of kpc) allow us to resolve the different galaxy components such as nuclei, bulges, disks, and spiral arms. Moreover, dwarf galaxies, by far the most common (yet still very poorly understood) galaxies in the universe, can only be observed locally.

A volume-limited sample was chosen as a way to limit distance dependent selection biases. To limit any possible contamination due to the cosmic variance, the volume should be much larger than the typical dimension of large-scale structures $(\sim 30 \mathrm{Mpc})$. At the same time it should be representative of the whole galaxy population inhabiting the local universe. A near-infrared $K$-band selection was adopted for two major reasons: (i) unlike optical surveys, which have strong selection effects due to the presence of dust, near-IR data are only marginally affected by dust extinction; (ii) whereas the optical flux is highly dependent on the number of relatively young stars and thus sensitive to recent episodes of star formation, the near-IR luminosity is a good measure of the overall stellar mass (e.g., Gavazzi et al. 1996), which recent studies suggest as the most important parameter in characterizing the statistical and evolutionary properties of galaxies. Indeed galaxy properties that appear to be tightly correlated with the galaxy's stellar mass include the following: physical properties, such as the star formation activity, the gas content and the metallicity (Boselli et al. 2001; 2002a; Zaritsky et al. 1994; Gavazzi et al. 2004; Tremonti et al. 2004); structural properties, such as the concentration index and the galaxy's light profile (Boselli et al. 1997; Gavazzi et al. 2000a; Scodeggio et al. 2002); the amount and distribution of dark matter, as indicated by the Tully-Fisher relation and the shape of the rotation curve for spirals (Tully \& Fisher 1977; Catinella et al. 2006) and the fundamental plane for ellipticals (Dressler et al. 1987; Djorgovski \& Davis 1987); the stellar population, shown through the color-magnitude relations for early-type (Visvanathan \& Sandage 1977, Bower et al. 1992) and late-type galaxies (Tully et al. 1982, Gavazzi et al. 1996) and the galaxy spectral energy distributions (Gavazzi et al. 2002b, Kauffmann et al. 2003). These correlations all indicate a down-sizing effect (e.g., Gavazzi et al. 1996; Cowie et al. 1996; Heavens et al. 2004), in which galaxies with high stellar masses formed most of their stars at a much earlier cosmic epoch than those with low stellar masses. This underline the dominant role of mass, rather than morphology, in shaping galaxies.

The final sample includes galaxies with a large range in luminosity (mass) and includes all Hubble types. Because of its definition, the selected sample also includes galaxies belonging to different density regions such as the core of the Virgo cluster, groups and pairs and isolated objects. Given its completeness, the Herschel Reference Sample (HRS) will be a suitable reference for any statistical study. Combined with KINGFISH (the Herschel extension of SINGS, Kennicutt et al. 2003) and VNGS (see $\S 3$, footnote 23), two samples optimized for the study of the different phases of the ISM in individual galaxies, these samples will provide the community with a unique dataset for studying the ISM properties of galaxies in the local universe.

The paper is organized as follows. Section 2 describes the scientific goals of the survey. Section 3 gives a description of the selection criteria used to define the sample. Section 4 gives details of the SPIRE observations we will carry out and $\S 5$ gives an overview of the multifrequency data that is available for the sample. Section 6 gives a brief description of the data processing and products. The multifrequency statistical properties of the sample will be described in $\S 7$.

\section{SCIENTIFIC OBJECTIVES}

The overall goal of this Herschel survey is to improve our knowledge of the cold dust properties of the most common extragalactic sources populating the local universe. Combined with multifrequency ancillary data covering the entire electro- 
magnetic spectrum (see $\S 5$ ), the new Herschel data will provide us with a unique data set with which to:

1. Trace, for the first time, the variation of the properties of the cold dust component (dust mass and temperature, dust to gas ratio,z...) of normal galaxies along the Hubble sequence, and as a function of luminosity. Given the large dispersion in galaxy properties (Morton \& Haynes 1994), it is important that the sample to be large enough to contain representatives of all galaxy types and include both early- and late-type galaxies spanning the largest possible range in luminosity. Where galaxies are resolved, we can analyze the distribution of the cold dust in the different galaxy components, e.g., the nuclei, bulges, spiral arms, and diffuse disks. This will provide important constraints on dust formation and evolution in galaxies (Galliano et al. 2008).

2. Study the role of dust in the physics of the ISM. As discussed in the introduction, dust plays a major role in the energetic equilibrium of the ISM. It acts as catalyst for the formation of the molecular hydrogen (Hollenbach \& Salpeter 1971; Duley \& Williams 1986) and shields the molecular gas component preventing dissociation from the diffuse interstellar radiation field (Hollenbach \& Tielens 1997). To understand the nature of the ISM we thus need to know how the cold dust properties (temperature, composition, geometrical distribution...) relate to other physical properties such as metallicity and interstellar radiation field (Boselli et al. 2004; Galliano et al. 2008). By combining dust surface brightnesses with metallicity dependent gas-to-dust ratios and HI column densities, submillimeter measurements can be used to determine $\mathrm{H}_{2}$ column densities (Guélin et al. 1993; 1995; Neininger et al. 1996; Boselli et al. 2002a). SPIRE data will therefore provide us with an independent measure of the molecular hydrogen component, and can be use for an accurate calibration of the still highly uncertain $\mathrm{CO}$ to $\mathrm{H}_{2}$ conversion factor.

3. Study the relationship between dust emission and star formation. Dust participates in the cooling of the gas through the shielding of the interstellar radiation field, in particular of the UV light, and thus plays a major role in the process of star formation (Draine 1978; Dwek 1986; Hollenbach \& Tielens 1997). The energy absorbed by dust is then radiated in the infrared domain. For the same reason dust emission has often been used as a tracer of star formation. We still do not know, however, what is the relationship between the cold dust emission and star formation. The study of resolved galaxies will allow us to analyze the relationship between the infrared surface brightness and the dust temperature (Chanial et al. 2007) and to trace the connections between the star formation and the dust and gas properties at galactic scales, extending the recent results of Spitzer to all phases of the ISM (Gordon et al. 2004, Calzetti et al. 2005, 2007; Perez-Gonzalez et al. 2006; Kennicutt et al. 2007, Prescott et al. 2007; Thilker et al. 2007).

4. Study the dust extinction properties in galaxies. Astronomers have tackled with the problem of dust opacity in galaxies for over 40 yr (e.g., Holmberg 1958; Disney et al. 1989; Calzetti 2001), but have reached no consensus. The key problem is that optical catalogs contain huge selection effects because of the existence of dust. We will be able to address this issue in two ways. First, the submillimeter images will, for the first time, allow us to determine the distribution of the dust column density in a very large number of galaxies. Second, we will be able to determine the global dust opacity in each galaxy by using the energy balance between the absorbed stellar light and the dust emitted radiation (Buat \& Xu 1996, Witt \& Gordon 2000, Buat et al. 2002, Cortese et al. 2006; 2008a). This requires an accurate determination of the UV to near-IR (stellar light) and midIR to submillimeter (dust emission) spectral energy distribution (Boselli et al. 2003a). By comparing the dust attenuation properties of different classes of objects, this analysis will allow us to define standard recipes for correcting UV and optical data, a useful tool for the interpretation of all modern surveys.

5. Determine whether there is an intergalactic dust cycle. Apart from the obvious possibility that dust is ejected from galaxies by the same methods that gas is ejected, such as through interactions with the surrounding environment (see point 9) and starburst-driven "superwinds" as indeed observed in M82 (Engelbracht et al. 2006), there is also the possibility that dust is ejected from galaxies by radiation pressure (Davies et al. 1998, Meiksin 2009, Oppenheimer \& Davé 2008). The ejection of dust from galaxies might explain the huge reservoir of metals found in the intergalactic medium (Rayan-Weber et al. 2006). There is clear evidence for dust in superwinds (Alton et al. 1999) and there is tantalizing evidence from ISO observations for extended distributions of dust around galaxies (Alton et al. 1998; Davies et al. 1999). Observations so far have been limited by either sensitivity (SCUBA) or resolution (ISO and Spitzer). The Herschel Reference Survey will be able to determine whether dust ejection is important because (i) we will be observing several hundred galaxies, so even if these phenomena are rare our sample should contain some examples, and (ii) our observations will have the sensitivity to detect dust well outside the optical disk of each galaxy.

6. Determine the amount of interstellar dust in ellipticals. Very little is known about dust in ellipticals. Despite the stereotype that ellipticals do not contain any dust, the structures seen in optical images imply that at least $50 \%$ of ellipticals contain some dust (Ferrarese et al. 2006). The amount of interstellar dust is too small, however, to be easily detected through its far-infrared emission; IRAS, for example, detected only about $15 \%$ of ellipticals (Bregman et al. 1998). Although Spitzer has now detected many ellipticals, these studies have mostly been of sources known a priori to contain some dust (Kaneda et al. 2007; Temi et al. 2007; Panuzzo et al. 2007) or molecular gas (Young et al. 2008). The SLUGS did contain a small statistically-complete sample of 11 ellipticals, although some of the six $850 \mu$ m detections may well have been of synchrotron rather than dust emission (Vlahakis et al. 2005). Other ellipticals 
have been detected at $350 \mu \mathrm{m}$ by Leeuw et al. (2008). The HRS contains 65 early-type (E, S0, and S0a) galaxies and our observations will have sufficient sensitivity to detect dust masses down to $\sim 10^{4} M_{\odot}$. The Herschel Reference Survey will therefore provide an unambiguous answer to the question of how much dust is in ellipticals.

7. Determine the origin of dust in ellipticals. The three possible origins of the dust in ellipticals are that (i) it has been produced in the atmospheres of the old evolved stars that dominate the light of ellipticals today, (ii) its origin is external to the elliptical and is the result of a merger, (iii) it is the relic of dust formed during the active star-forming phase early in the history of the galaxy. The distribution of dust is an important clue to understand its true origin. The presence of the first mechanism is suggested by Spitzer mid-infrared spectra of Virgo ellipticals (Bressan et al. 2006) which show the silicate emission produced by dust in circumstellar envelopes of evolved stars. The origin of this feature (predicted by Bressan et al. 1998) is supported by the finding that the mid-infrared emission has the same profile as optical light (Xilouris et al. 2004) in many early-type galaxies. It is unclear, however, whether this is the predominant mechanism producing the interstellar dust in these galaxies. For example, a submillimeter map of the nearby elliptical Centaurus A, has revealed a dusty disk, implying that the dust (and the galaxy itself) has been formed as the result of a merger (Leeuw et al. 2002). Temi et al. (2007) also found little correlation between the Spitzer 70 and $160 \mu \mathrm{m}$ emission and optical starlight, which also suggests the dust has an external origin.

We will use the Herschel Reference Survey results to investigate the origin of the dust by, first, investigating the detailed morphology of the dust, in particular how it compares with the stellar distribution, and, second, by investigating whether the mass of dust is correlated with other global properties of the galaxy such as stellar mass. It has often been argued that sputtering by the ubiquitous, hot X-ray emitting gas in early galaxies should destroy any dust formed more than $10^{8}$ years in the past (Tielens et al. 1994). However, the fact that dust is seen visually in 50\% of ellipticals (Ferrarese et al. 2006) and the recent discovery of small grains, which are preferentially destroyed by sputtering, in ellipticals (Kaneda et al. 2007) suggests that dust is protected in some way. We will investigate whether sputtering from the hot gas destroys the dust grains and will test the internal origin hypothesis by investigating any possible correlation between $\mathrm{X}$-ray excess and dust mass.

8. Measure the local luminosity and dust-mass distributions. These distributions are important benchmarks for the deep Herschel surveys. These have already been estimated as part of SLUGS (Dunne et al. 2000; Vlahakis et al. 2005): the much greater sensitivity of the Herschel Reference Survey will push these limits down by a factor of $\sim 50$.

9. Understand the role of the environment on the evolution of galaxies. The well-known phenomenon that spiral galaxies in clusters are HI-deficient and have truncated HI disks (e.g., Cayatte et al. 1990) demonstrates that the environment of a galaxy can have a strong effect on its interstellar medium. Indeed there is clear evidence for tidally-stripped dust in interacting galaxies (Thomas et al. 2002) and indications for ram-pressure stripped dust in cluster objects (Boselli \& Gavazzi 2006). At the same time the presence of metals (Sarazin 1986) and possibly of dust (Stickel et al. 2002; Montier \& Giard 2005) in the diffuse intracluster medium has been shown by X-ray and far-IR observations. The comparison of the submillimeter emission of cluster and isolated galaxies within the HRS will allow us to make a detailed study on the effects of the environment on the dust properties of galaxies, and thus understand whether the hot and dense cluster intergalactic medium can be polluted through the gas stripping process of late-type galaxies (Boselli \& Gavazzi 2006).

10. Provide a multifrequency reference sample suitable for any statistical study. Our aim is that the HRS will be the first large sample of galaxies with observations of all phases of the ISM, as well as measurements over the entire wavelength range of the spectral energy distribution (SED) for each galaxy. The sample will then serve for many purposes; e.g., the UV to radio continuum SEDs could, for example, be used to determine the nature of unresolved sources or as templates for estimating photometric redshifts.

\section{THE SAMPLE}

The Herschel Reference Sample (HRS) has been selected according to the following criteria:

1. Volume-limited: A volume limit was imposed to reduce distance uncertainties due to local peculiar motions and ensure the presence of low-luminosity, dwarf galaxies, not accessible at high redshift. By applying a lower distance $(D)$ limit we exclude the very extended sources, the observing of which would be too time consuming ${ }^{23}$. We have selected all galaxies with an optical recessional velocity (vel, taken from NED) in between $1050 \mathrm{~km} \mathrm{~s}^{-1}$ and $1750 \mathrm{~km} \mathrm{~s}^{-1}$ that, for $\mathrm{H}_{0}=70 \mathrm{~km} \mathrm{~s}^{-1}$ $\mathrm{Mpc}^{-1}$ and in the absence of peculiar motions, corresponds to $15 \leq D \leq 25 \mathrm{Mpc}^{24}$. In the Virgo cluster region $\left(12^{\text {h }}<\right.$ R.A. $(2000)<13^{\text {h }} ; 0^{\circ}<$ decl. $\left.<18^{\circ}\right)$, where peculiar motions are dominant, we have included all galaxies with vel $<$ $3000 \mathrm{~km} \mathrm{~s}^{-1}$ and belonging to cluster A, the North (N) and East (E) clouds, and the Southern extension (S) (17 Mpc) and Virgo B $(23 \mathrm{Mpc})$, where the subgroup membership has been taken from Gavazzi et al. (1999a). W and M clouds objects, at a distance of $32 \mathrm{Mpc}$, have been excluded. ${ }^{25}$

\footnotetext{
${ }^{23} \mathrm{~A}$ small sample of very nearby and extended galaxies will be observed in detail as part of another guaranteed time project: Physical Processes in the Interstellar Medium of Very Nearby Galaxies.

${ }^{24}$ Given the possible discrepancy between optical and $\mathrm{HI}$ recessional velocity measurements, heliocentric velocities given in Table 1 can be outside this range.
} 

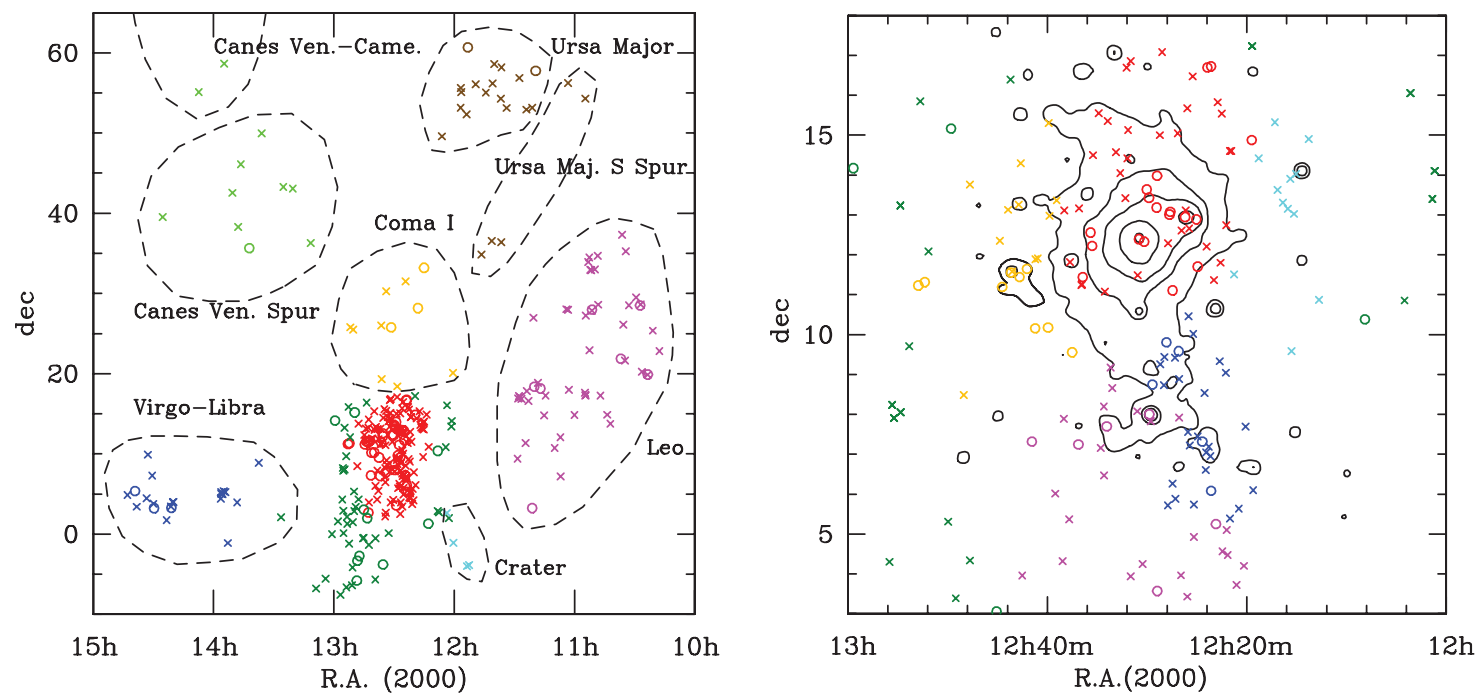

FIG. 1.-Sky distribution of the HRS for early-type (E-S0-S0a; circles) and late-type (Sa-Sd-Im-BCD; crosses) galaxies (left panel). Dashed contours delimit the different clouds. The large concentration of galaxies in the center of the figure is the Virgo cluster (red) with its outskirts (dark green) Orange symbols are for Coma I Cloud, magenta for Leo Cloud, brown for Ursa Major Southern Spur and Cloud, cyan for Crater Cloud, light green for Canes Venatici Spur and Camelopardalis, and blue for Virgo-Lybra Cloud galaxies, respectively. The Virgo cluster region (right panel): black contours show the diffuse X-ray emission of the cluster (from Böhringer et al. 1994). Red symbols are for galaxies belonging to the Virgo A cloud, blue to Virgo B, orange to Virgo E, magenta to Virgo S, cyan to Virgo N, and dark green to the Virgo outskirts, as defined by Gavazzi et al. (1999a).

2. $K$ band selection: Given the expected low dust content of quiescent galaxies, whose emission would be hardly detectable within reasonable integration times, a more stringent limit has been adopted for early-types than for star-forming galaxies. Among those galaxies in the required recessional velocity range, we thus selected all late-type spirals and irregulars (Sa-Sd-Im-BCD) with a 2MASS $K$ band total magnitude $K_{\text {Stot }} \leq 12$ and all ellipticals and lenticulars (E, S0, S0a) with $K_{\text {Stot }} \leq 8.7$.

3. High galactic latitude: To minimize galactic cirrus contamination, galaxies have been selected at high galactic latitude $\left(b>+55^{\circ}\right)$ and in low galactic extinction regions $\left(A_{B}<0.2\right.$; Schlegel et al. 1998).

The resulting sample is composed of 323 galaxies located in the sky region between $10^{\mathrm{h}} 17^{\mathrm{m}}<$ R.A. $(2000)<14^{\mathrm{h}} 43^{\mathrm{m}}$ and $-6^{\circ}<$ decl. $<60^{\circ}$ (see Fig. 1), of which 65 are early-type (E, S0 and S0a) and 258 are late-type (Sa-Sd-Im-BCD) (see Fig. 2). Figure 2 shows that the only galaxies which are clearly undersampled are blue compact galaxies and dwarf irregulars, the most numerous galaxies in the nearby universe. They will be the targets of another SPIRE key program. ${ }^{26}$ As selected, the

\footnotetext{
${ }^{25}$ The sample was selected before distances were available on NED. According to these new NED estimates, the distance of the HRS galaxies outside the Virgo cluster are generally included in the $15 \leq D \leq 25 \mathrm{Mpc}$ range. In the Virgo region, however, our distances are determined according to subgroup membership criteria, and are thus generally different than those given by NED.

${ }^{26}$ The ISM in Low-Metallicity Environments: Bridging the Gap Between
}

sample spans a large range in environment since it includes the Virgo cluster, many galaxy groups and pairs as well as relatively isolated objects (Fig. 1). Using the Virgo cluster membership criteria defined in Gavazzi et al. (1999a), the HRS includes 82 members of cluster A and B (Fig. 2) The other galaxies are members of nearby clouds such as Leo, Ursa Major and Ursa Major Southern Spur, Crater, Coma I, Canes Venatici Spur and Canes Venatici-Camelopardalis and Virgo-Libra Clouds (Tully 1988). As defined, the sample is thus ideal for environmental studies (Cortese \& Hughes 2009; Hughes \& Cortese 2009). The whole sample is given in Table 1 with columns arranged as follows:

Column 1: Herschel Reference Sample (HRS) name. This is the position of the galaxy in the sample list when sorted by increasing right ascension.

Column 2: Zwicky name, from the Catalogue of Galaxies and of Cluster of Galaxies (CGCG; Zwicky et al. 1961-1968).

Column 3: Virgo Cluster Catalogue (VCC) name, from Binggeli et al. (1985).

Column 4: Uppsala General Catalog (UGC) name (Nilson 1973).

Column 5: New General Catalogue (NGC) name.

Column 6: Index Catalogue (IC) name.

Column 7: J2000 right ascension, taken from NED.

Column 8: J2000 declinations, taken from NED.

Column 9: Morphological type, taken from NED, or from

Local Universe and Primordial Galaxies 


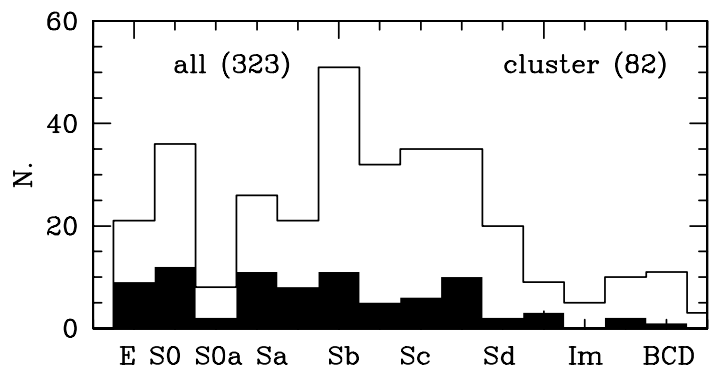

FIG. 2.-Distribution in morphological type of the HRS for all (empty histogram) and cluster (filled histogram) galaxies. The cluster sample is composed of galaxies members of the Virgo A and B clouds.

our own classification if not available.

Column 10: Total $K$ band magnitude $\left(K_{\text {Stot }}\right)$, from 2MASS (Skrutskie et al. 2006).

Column 11: Optical isophotal distance D 25 (25 mag $\operatorname{arcsec}^{-2}$ ), from NED.

Column 12: Heliocentric radial velocity (in $\mathrm{km} \mathrm{s}^{-1}$ ), from HI data when available, otherwise from NED.

Column 13: Cluster or cloud membership, from Gavazzi et al. (1999a) for Virgo, and Tully (1988) or Nolthenius (1993) whenever available, or from our own estimate otherwise.

Column 14: Pair/group membership, from Karachentsev et al. (1972) or from NED whenever available, or from our own estimate elsewhere. Pair/group membership has been assigned according to the following criteria: close pairs (CP) are those with a nearby companion at a distance less than $50 \mathrm{kpc}$ and a difference in redshift $<600 \mathrm{~km} \mathrm{~s}^{-1}$, as in Gavazzi et al. (1999b), while pairs (P) up to $150 \mathrm{kpc}$. The number indicates whether the galaxy belongs to a triplet (3), quadruplet (4), and quintuplet (5). Groups outside Virgo and its immediate surroundings have been determined by counting the number of bright galaxies ${ }^{27}$ within $25^{\prime}$ (which, at a median distance of $20 \mathrm{Mpc}$, corresponds to $\sim 150 \mathrm{kpc}$ ) and $600 \mathrm{~km} \mathrm{~s}^{-1}$. Pairs in the Virgo region are only those catalogued by Karachentsev et al. (1972).

Column 15: Galactic extinction $A_{B}$ (Schlegel et al. 1998).

Column 16: Alternative names.

\section{SPIRE OBSERVATIONS}

With its large field of view $\left(4^{\prime} \times 8^{\prime}\right)$, its sensitivity ( $S \sim 7 \mathrm{mJy}, 5 \sigma$ in $1 \mathrm{hr}$, point source mode), and angular resolution ( $\sim 30$ arcsec; see Table 2), SPIRE on Herschel is the ideal instrument for the proposed survey.

In constructing our observing program, we have used different integration times for early- and late-type galaxies because the former are known to contain less dust.

Integration times for early-types were determined by assuming a lower limit of $\sim 10^{4} M_{\odot}$ to their total dust mass as deter-

\footnotetext{
${ }^{27}$ For bright galaxies, we intend those included in major catalogs such as NGC, UGC, IC, or CGCG.
}

mined from IRAS observations (Bregman et al. 1998) or measured from optical absorption line measurements (Goudfrooij et al. 1994; Van Dokkum \& Franx 1995; Ferrari et al. 1999). At $20 \mathrm{Mpc}$, a galaxy with a dust mass of $\sim 10^{4} M_{\odot}$ would have a flux density at $250 \mu \mathrm{m}$ of $11 \mathrm{mJy}$. We estimate that with the adopted integration times we should detect an elliptical with $\sim 10^{4} M_{\odot}$ of dust at the $3 \sigma$ level.

Integration times for spirals have been chosen so that we should be able to detect dust outside the optical radius, ${ }^{28}$ for which there is evidence from ISO observations (Alton et al. 1998; Davies et al. 1999). By combining ISOPHOT (Alton et al. 1998) and SCUBA (Vlahakis et al. 2005) observations of extended sources with the spectral energy distribution of normal galaxies of different type (Boselli et al. 2003a), and assuming a standard gas-to-dust ratio of $\sim 100$ with the extragalactic calibration for the dust-emissivity coefficient (James et al. 2002), we estimate that the dust associated with the extended HI disk would have a flux density of $\sim 14 \mathrm{mJy}$ beam $^{-1}$ at $250 \mu \mathrm{m}$ for $\mathrm{HI}$ column densities of $\sim 10^{20}$ atoms $\mathrm{cm}^{-2}$. In normal galaxies such gas column densities are generally reached well outside the stellar disk, at $\sim 1.5$ optical radii (Cayatte et al. 1994; Broelis \& Van Woerden 1994). Because of metallicity gradients, however, the gas-to-dust ratio is expected to increase in the outer disks, as observed in M31 by Cuillandre et al. (2001). We would therefore expect to observe lower far-IR flux densities. With $12 \mathrm{~min}-$ utes of integration time ( 3 scan-maps) we will get to an instrumental noise of $6.9 \mathrm{mJy}^{\text {beam }}{ }^{-1}$, when the expected emission is $14 \mathrm{mJy}_{\text {beam }}{ }^{-1}$; this sensitivity will allow us to detect the dust emission in the outer disk by integrating flux densities along elliptical annuli with a sensitivity of $\sim 30$ better than that of previous observations (Alton et al. 1998).

All galaxies outside the Virgo cluster will be observed in scan-map mode $\left(30^{\prime \prime} \mathrm{s}^{-1}\right)$. For each galaxy, the size of the scan-map has been chosen based on the optical size of the galaxy. For late-type galaxies, the observing mode has been chosen so that our images will cover the galaxy at least out to $1.5 \times D_{25}$ (where $D_{25}$ is the $25 \mathrm{mag} \operatorname{arcsec}^{-2}$ isophotal diameter) i.e. out to the approximate $\mathrm{HI}$ radius. For spirals we will make three pairs of cross-linked observations, which will reach a $1 \sigma$ sensitivity in instrumental noise of $6.9,4.6$, and $6.9 \mathrm{mJy}_{\text {beam }}^{-1}$ at 250,350 , and $500 \mu \mathrm{m}$, respectively as determined during the science verification phase. These values are comparable to the noise from the confusion of faint unrelated sources $(4,5$, and $6 \mathrm{mJy}_{\text {beam }}^{-1}$ in the three bands). Elliptical galaxies are likely to be weaker and thus need longer integration times, we have compromised by only requiring the image to contain the galaxy out to $D_{25}$. For ellipticals and S0s we will make eight pairs of cross-linked observations reaching a $1 \sigma$ sensitivity in instrumental noise of $4.2,2.8$, and $4.2 \mathrm{mJy}$ beam $^{-1}$ at 250 ,

\footnotetext{
${ }^{28}$ Defined as the $B$-band isophotal radius at $25 \mathrm{mag} \operatorname{arcsec}^{-1}$
} 


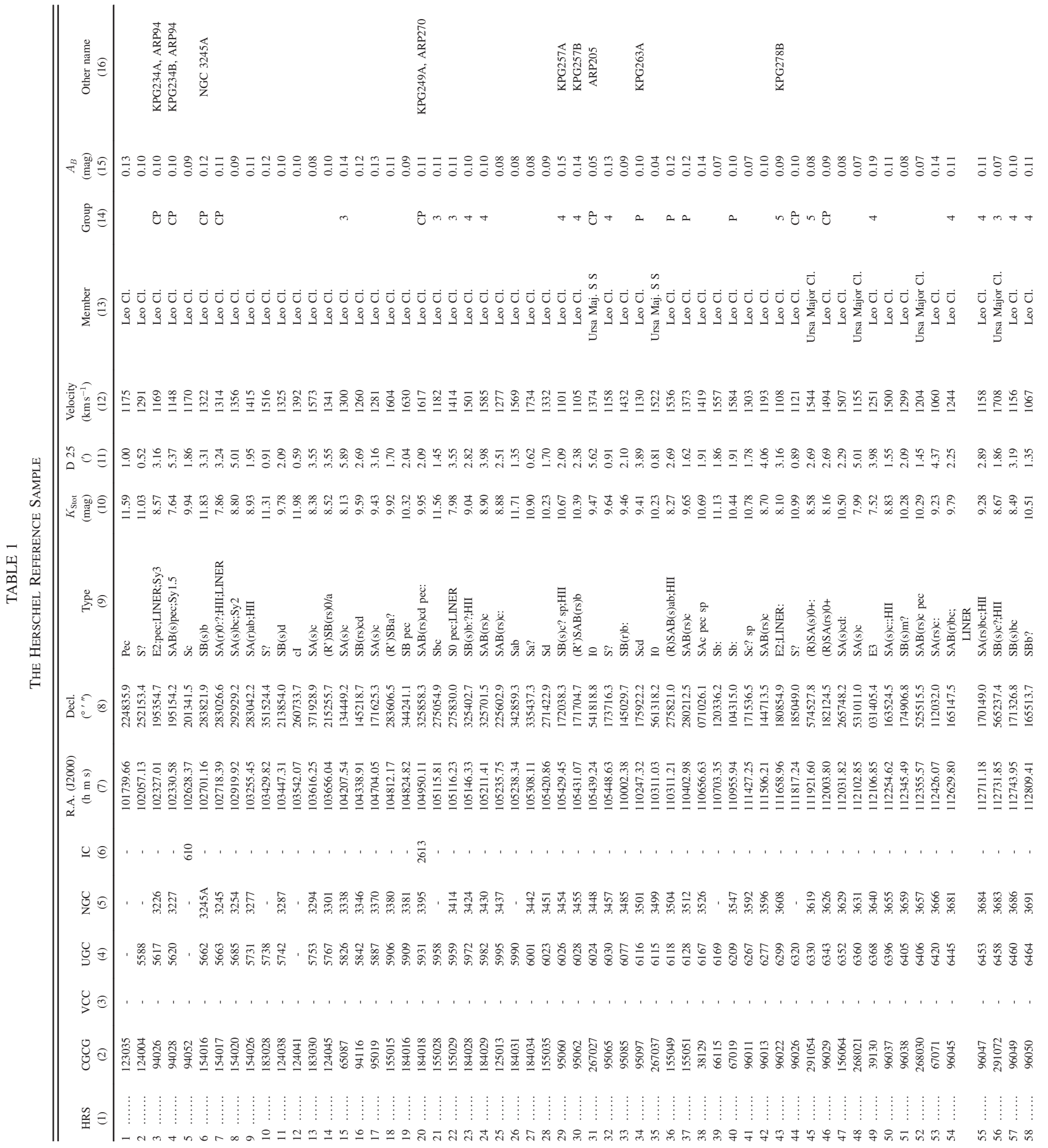




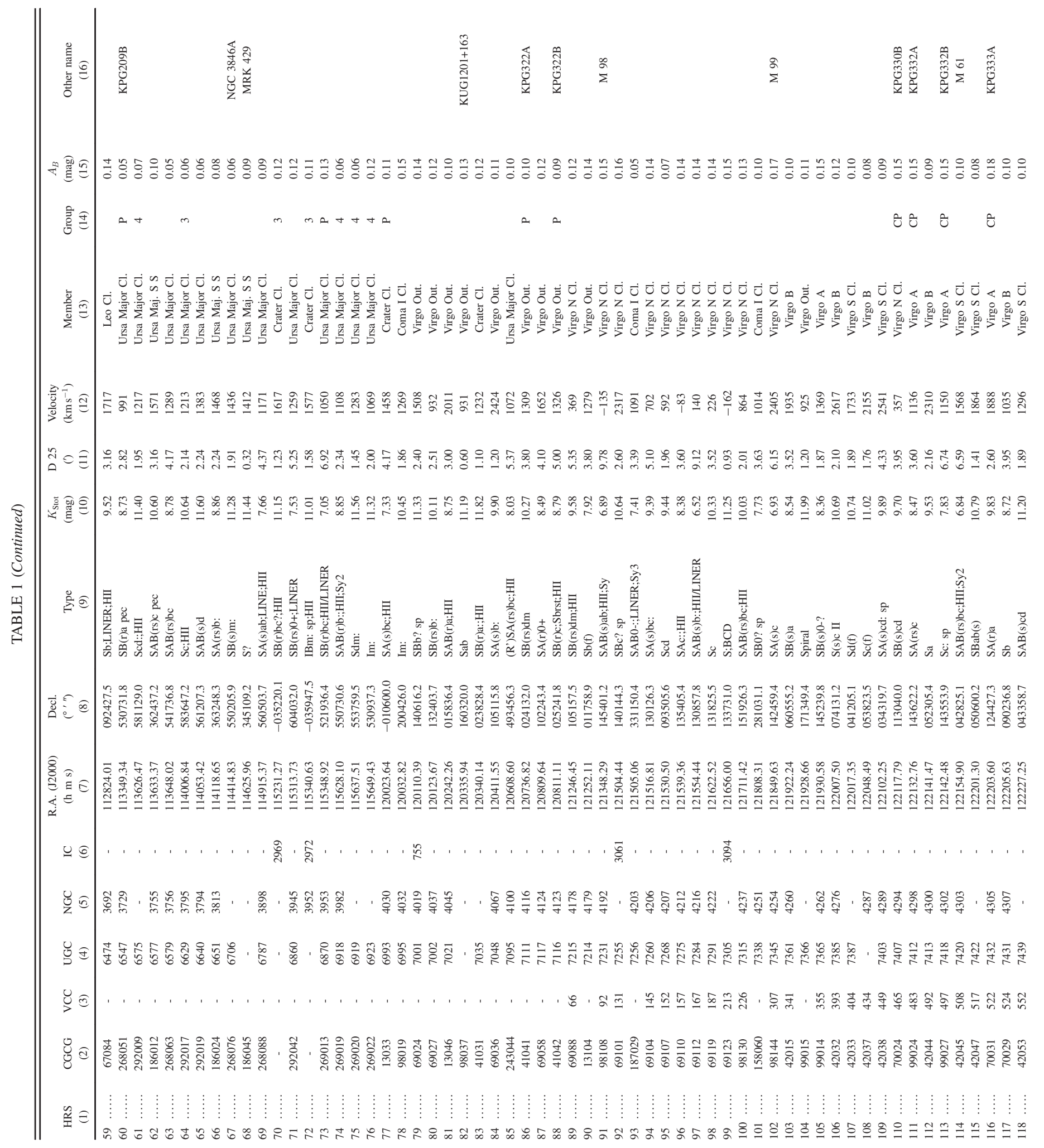




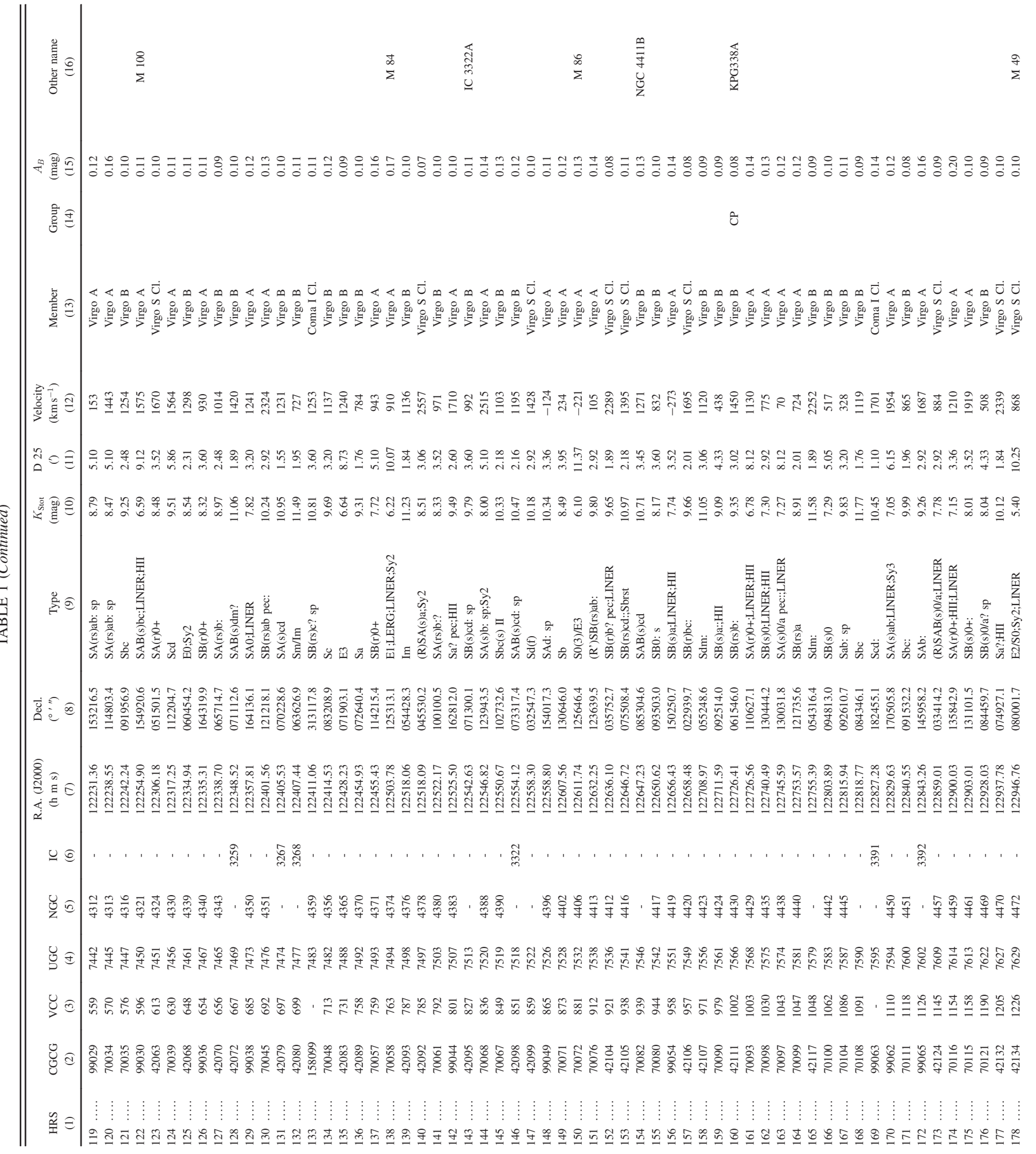




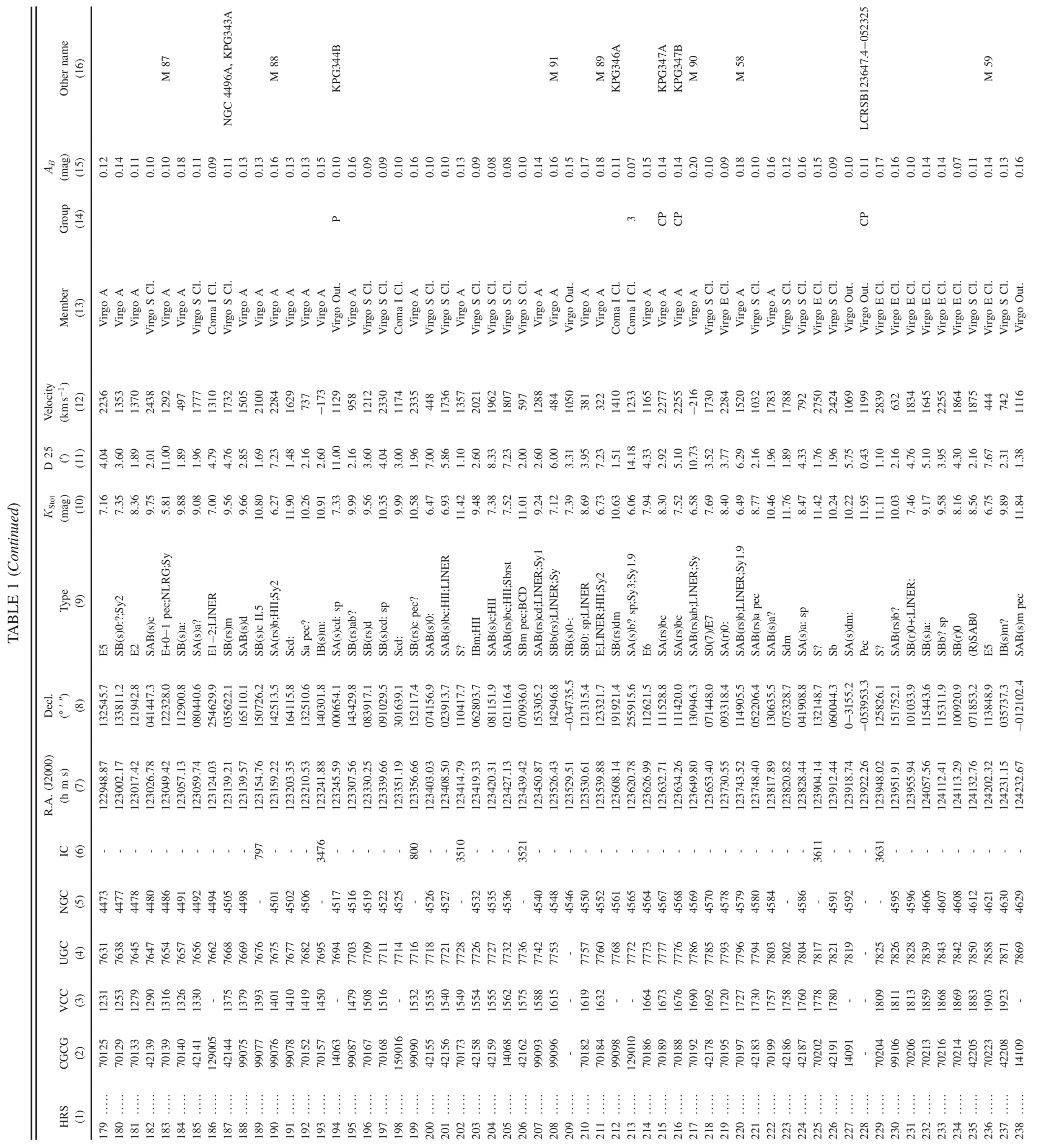




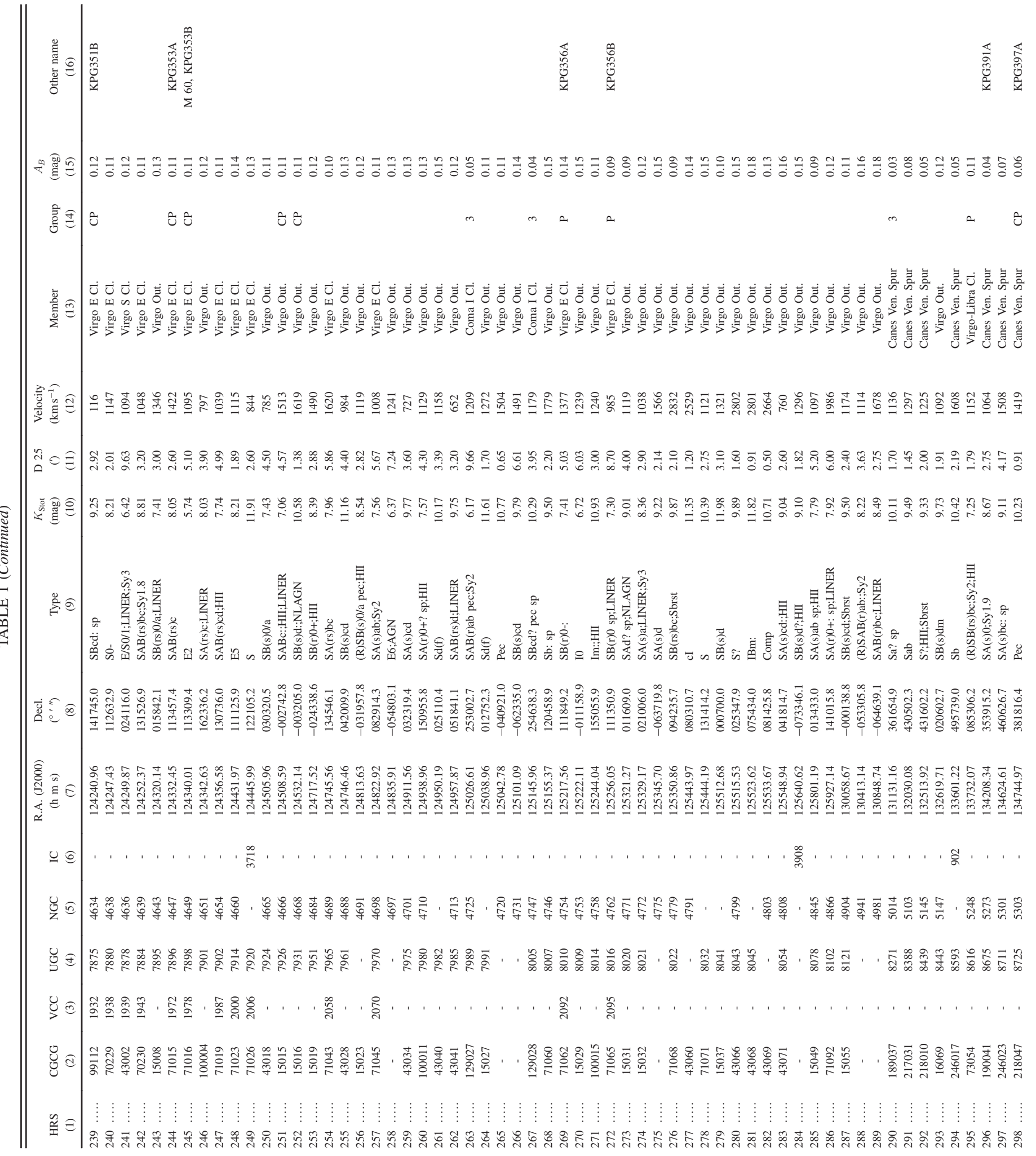




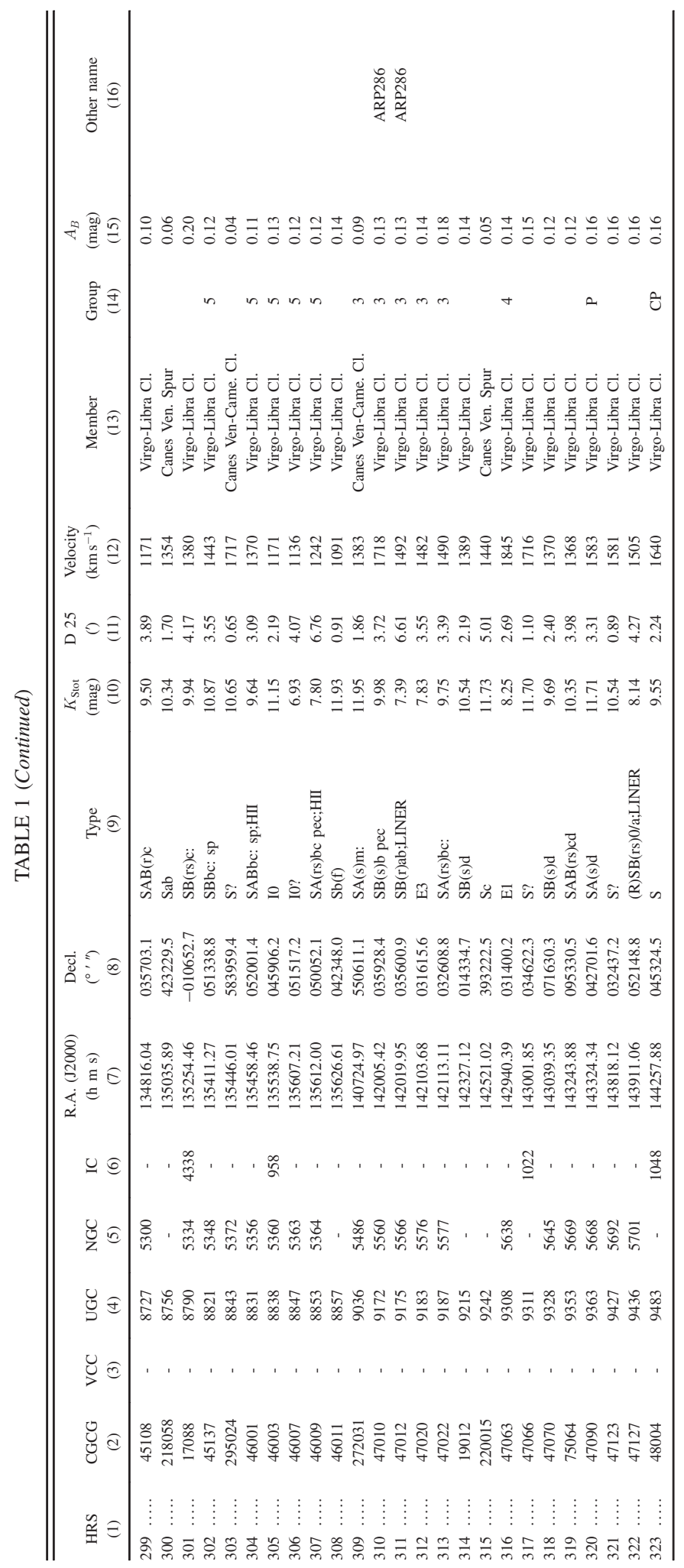


TABLE 2

SPIRE PERFORMANCE

\begin{tabular}{rcccc}
\hline \hline$\lambda$ & & $250 \mu \mathrm{m}$ & $350 \mu \mathrm{m}$ & $500 \mu \mathrm{m}$ \\
\hline Ang. Res. & $\ldots$. & $18.1^{\prime \prime}$ & $25.2^{\prime \prime}$ & $36.9^{\prime \prime}$ \\
Lin. Res. & $\ldots \ldots$ & $1.7 \mathrm{kpc}$ & $2.4 \mathrm{kpc}$ & $3.6 \mathrm{kpc}$ \\
Sensitivity & $\ldots$. & 12 mJy beam $^{-1}$ & 8 mJy beam $^{-1}$ & 12 mJy beam $^{-1}$ \\
\hline
\end{tabular}

NoTE. $-1 \sigma$ sensitivity (instrumental noise) for a standard map (one repeat $=$ two crosslinked scans) as determined during the science verification phase (onboard observations). The linear resolution is at $20 \mathrm{Mpc}$.

350 , and $500 \mu \mathrm{m}$, respectively, which in the latter two bands is below the noise expected from the confusion of faint sources.

Sixty square degrees centered on the Virgo cluster region will be covered by Herschel in parallel mode (i.e. using both SPIRE and PACS) as part of the Herschel Virgo Cluster Survey HeViCS ${ }^{29}$ Eighty-three HRS galaxies fall into this region. To avoid duplication, these galaxies will be observed only during the HeViCS survey. HeViCS will survey this region in fast-scan mode $\left(60^{\prime \prime} \mathrm{s}^{-1}\right)$ using both SPIRE and PACS. The survey will make eight scans reaching a $1 \sigma$ of instrumental noise of 4.5, 6.2, and $5.3 \mathrm{mJy}_{\text {beam }}^{-1}$ at 250,350 , and $500 \mu \mathrm{m}$, respectively, as determined using the Herschel Observation Planning Tool (HSpot ver. 4.2.0). This is not as deep as our observations of ellipticals and S0s outside Virgo, but the effect of confusion noise means that the decrease in effective sensitivity is really small. On the plus side, for the HRS galaxies in Virgo we will also have PACS images at 110 and $160 \mu \mathrm{m}$.

\section{COMPLEMENTARY DATA}

A number of key aims of our survey (see $\S 2$ ) can only be achieved through the combination of Herschel observations with corollary data. UV to near-IR imaging is needed to determine the distribution and content of the different stellar populations, to quantify the intensity of the interstellar radiation field and to study the recent activity of star formation (UV and $\mathrm{H} \alpha$ ). Optical spectroscopy is crucial to measure stellar and gas metalicities and to determine the presence of any nuclear activity. At the same time, the Balmer emission line can be used to measure the dust extinction within HII regions and are thus essential for quantifying the current level of star formation activity. HI and $\mathrm{CO}$ line data are necessary for determining the content and distribution of the gaseous component of the ISM, the principal feeder of star formation in galaxies. High resolution HI imaging can also be used to study the kinematical properties of the target galaxies. Mid-, far-IR and submillimeter data, combined with SPIRE data, will be used to study the physical properties of the different dust components (PAHs, very small grains, and large grains), and radio continuum data for measuring the thermal and synchrotron emission.

\footnotetext{
${ }^{29}$ At www.arcetri.astro.it/hevics.
}

Given its definition, the HRS is easily accessible for groundbased and space facilities: the selected galaxies are in fact relatively bright $\left(m_{B}<15 \mathrm{mag}\right)$ and extended $(\sim 2-3 \mathrm{arcmin})$. Listed in this section are the most important references for ancillary data.

\subsection{UV, Optical, Near-IR, and $\mathrm{H} \alpha$ Imaging}

Of the 323 galaxies in the HRS, 280 have been observed by the Galaxy Evolution Explorer (GALEX) in the two UV bands FUV $\left(\lambda_{\text {eff }}=1539 \AA, \Delta \lambda=442 \AA\right)$ and NUV $\left(\lambda_{\text {eff }}=2316 \AA\right.$, $\Delta \lambda=1069 \AA)$. Observations have been taken as part of the Nearby Galaxy Survey (NGS; Gil de Paz et al. 2007), the Virgo cluster survey (Boselli et al. 2005), the All Imaging Survey (AIS) or as pointed observations in open time proposals. A GALEX legacy survey has been recently accepted to complete the UV observation of the HRS galaxies at a deepness of the Medium Imaging Survey (1500 s per field).

SDSS (DR7 release, Adelman-McCarthy et al. 2008) images in the ugriz filters are available for 313 objects. All galaxies have been observed in the $J H K$ filters by 2MASS (Jarrett et al. 2003). Deep $B, V, H$, and $K$ band images for all Virgo cluster galaxies and for some Coma I Cloud galaxies are available on the GOLDMine database ${ }^{30}$ (Gavazzi et al. 2003). These have been taken with pointed observations during the near-infrared and optical extensive surveys of the Virgo cluster carried out by Boselli et al. (1997, 2000, 2003b) and Gavazzi et al. (2000b).

An $\mathrm{H} \alpha+[\mathrm{NII}]$ imaging survey of the star-forming HRS galaxies found outside the Virgo cluster under way at the $2.1 \mathrm{~m}$ San Pedro Martir telescope is almost complete. Combined with images taken in the Virgo cluster region (Boselli \& Gavazzi 2002, Boselli et al. 2002b; Gavazzi et al. 1998; 2002a; 2006) $\mathrm{H} \alpha+$ [NII] data are now available for 221 of the 258 late-type objects and 26 of the 65 early-types.

\subsection{Integrated Spectroscopy}

A low resolution $(R \sim 1000)$, integrated spectroscopic survey in the wavelength range $3500-7200 \AA$ is under way at the $1.93 \mathrm{~m}$ OHP telescope. In order to sample the spectral properties of the whole galaxy, and not just those of the nuclear regions (these last provided by the SDSS for 106 galaxies in the DR6), observations have been executed using the drifting technique described in Kennicutt (1992). Exposures are taken while constantly and uniformly drifting the spectrograph slit over the full extent of the galaxy. A resolution $R \sim 1000$ is mandatory for resolving [NII] from $\mathrm{H} \alpha$ and measuring the stellar underling Balmer absorption under $\mathrm{H} \beta$.

Data for 64 Virgo cluster galaxies in the HRS have already been published in Gavazzi et al. (2004) along with a few other galaxies in Moustakas \& Kennicutt (2006), Jansen et al. (2000) and Kennicutt (1992b). We have data from the literature or from

\footnotetext{
${ }^{30}$ At http://goldmine.mib.infn.it/; Gavazzi et al. 2003
} 
our own observations for $256 / 258$ of the late-types and $33 / 65$ of the early-types, making the whole sample complete at $89 \%$. The remaining objects will be included in the future observing runs.

\subsection{HI and CO Lines}

Single-beam HI observations are available from a large variety of sources. Most of the galaxies have HI data in Springob et al. (2005) and Gavazzi et al. (2005), this last reference limited to the Virgo cluster region. Out of the 8 late-type galaxies, 249 have an $\mathrm{HI}$ measurements, as do 55/65 of the early-types. All galaxies in the $0^{\circ}<$ decl. $<30^{\circ}$ range $(80 \%)$ will be observed during the ALFALFA survey under way at the $305 \mathrm{~m}$ Arecibo radio telescope (Giovanelli et al. 2005). Data will be gathered with an homogeneous sensitivity $(\mathrm{rms}=2.5 \mathrm{mJy})$ and spectral resolution $\left(5.5 \mathrm{~km} \mathrm{~s}^{-1}\right)$ : at the average distance of the HRS the ALFALFA survey will detect all sources with $\mathrm{M}_{\mathrm{HI}} \geq 10^{7.5} M_{\odot}$. VLA and WSRT HI maps are available for 236 HRS galaxies, from the WHISP (van der Hulst 2002) and VIVA (Chung et al. 2009) survey, this last limited to the Virgo region, from VLA archives or from our own WSRT observations.

A ${ }^{12} \mathrm{CO}(1-0)$ line $(2.6 \mathrm{~mm}, 115 \mathrm{GHz})$ survey of the HRS galaxies is under way at the $12 \mathrm{~m}$ Kitt Peak telescope. Fiftyeight spiral galaxies have been observed to date, with an average rms of $3 \mathrm{mK}$ in $T_{R}^{*}$ scale. Thanks to these new observations and to data in the literature, $\mathrm{CO}$ measurements are now available for $161 / 258$ late-type and $22 / 65$ early-type galaxies, with a detection rate of $83 \%$ and $55 \%$, respectively. Data have been taken from different sources, mostly from the FCRAO survey of Young et al. (1995), or Kenney \& Young (1988) and Boselli et al. $(1995 ;$ 2002a) for the Virgo cluster region. We obtained time at the James Clerk Maxwell Telescope (JCMT) on the $345 \mathrm{GHz}$ HARP array receiver to search for $\mathrm{CO}(3-2)$ emission from the central $3^{\prime} \times 3^{\prime}$ region (with $15^{\prime \prime}$ resolution) of a subsample of late-type galaxies with $K_{\text {Stot }}<8.7$. As of the beginning of 2010 January, 42 of a total of 56 galaxies in the subsample have been observed to completion.

\subsection{Mid-IR, Far-IR, and Submillimeter}

Infrared data for the HRS galaxies are already available from different sources. 208/258 late-type and 32/65 of the early-type galaxies have been detected by IRAS at 60 and $100 \mu \mathrm{m}$, only 103 (Sa-Sd-Im-BCD) and 17 (E-S0-S0a) at 12 and $25 \mu \mathrm{m}$. Virgo galaxies have been observed with CAM (45), PHOT (26), and LWS (21) on ISO (Leech et al. 1999; Malhotra et al. 2001; Roussel et al. 2001; Boselli et al. 2002c, 2003b; Tuffs et al. 2002). Spitzer observations have been completed for 157 HRS galaxies with IRAC and 181 galaxies with MIPS. The data for all of these galaxies will eventually be available from the Spitzer archives. The pipeline-processed IRAC data are sufficient for science analysis, but MIPS data have been re- processed using the MIPS Data Analysis Tools (Gordon et al. 2005) along with additional processing software.

All HRS galaxies have been observed during the recently completed AKARI all sky survey in the 9, 20, 70, 90, and $160 \mu \mathrm{m}$ bands which covered $\sim 95 \%$ of the whole sky with an angular resolution comparable to that of SPIRE $\left(\sim 30^{\prime \prime}\right.$ at $90 \mu \mathrm{m})$ and a sensitivity $\sim 3$ times better than IRAS. Pointed observations with AKARI and SCUBA are also available of a few ellipticals.

\subsection{Radio Continuum Data}

The HRS galaxies have been observed by the NVSS survey at $20 \mathrm{~cm}(1415 \mathrm{MHz})$ (Condon et al. 1998) with an angular resolution of $45^{\prime \prime}$ and a sensitivity of $2.5 \mathrm{mJy}$. Twenty-two of 65 and $159 / 258$ of the early- and late-type galaxies, respectively, have been detected at $20 \mathrm{~cm}$. The data of the FIRST survey, at 5" resolution, are also available (Becker et al. 1995). For a fraction of galaxies, data at $6.3 \mathrm{~cm}$ and $2.8 \mathrm{~cm}$ are also available from Niklas et al. (1995) and Vollmer et al. (2004).

\subsection{X-ray data}

$\mathrm{X}$-ray data at $0.2-4 \mathrm{keV}$ from the Einstein Observatory imaging instruments (IPC and HRI) are available for 31 early-type and 52 late-type galaxies from Fabbiano et al. (1992) and Shapley et al. (2001). Ten HRS galaxies have been detected by the ROSAT all sky survey (Voges et al. 1999), while Chandra observations of many of the early-type objects within the HRS are still under way (Gallo et al. 2008).

Table 3 gives the complete sample at different wavelengths, while Table 4 shows the availability of ancillary data for the HRS galaxies.

\section{HRS DATA PROCESSING, DATA PRODUCTS, AND WEB SITE}

The HRS galaxies are distributed widely across the sky, the survey will progress along with the Herschel mission, that is expected to last for three and a half years, with the first six months for commissioning, performance verification, science demonstration and the last three years for science. Data for each single observation will be proprietary for one year after it is taken. During this time, the data will be processed using the most up-to-date pipeline developed by the SPIRE-Instrument Control

TABLE 3

Completeness of the Data SAMPle

\begin{tabular}{lccc}
\hline \hline & & E+S0+S0a & Sa-Sm-Im-BCD \\
\hline $\mathrm{K} \ldots \ldots \ldots \ldots \ldots \ldots \ldots \ldots$ & $100 \%$ & $100 \%$ \\
$\mathrm{HI} \ldots \ldots \ldots \ldots \ldots \ldots \ldots \ldots$ & $85 \%(40 \%)$ & $97 \%(93 \%)$ \\
FIR $(60 \mu \mathrm{m}) \quad \ldots \ldots \ldots \ldots \ldots$ & $95 \%(62 \%)$ & $89 \%(83 \%)$ \\
Radio cont. $(20 \mathrm{~cm}) \ldots \ldots$ & $100 \%(34 \%)$ & $100 \%(62 \%)$ \\
\hline
\end{tabular}

NoTE.-Values in parentheses give the detection rate. 
TABLE 4

Ancillary Data for the Herschel Reference Sample.

\begin{tabular}{|c|c|c|c|c|c|c|c|c|c|c|}
\hline HRS & & 2MASS & SDSS & UV & $\mathrm{H} \alpha$ & IRAS & $20 \mathrm{~cm}$ & $\mathrm{HI}$ & $\mathrm{CO}$ & Spec \\
\hline 1 & $\ldots \ldots$ & $X$ & $X$ & - & - & $X$ & - & - & - & $X$ \\
\hline $2 \ldots$ & $\ldots \ldots$ & $X$ & $\mathrm{X}$ & $\mathrm{X}$ & - & $\mathrm{X}$ & $\mathrm{X}$ & $X$ & - & $X$ \\
\hline $3 \ldots$ & $\ldots \ldots$ & $X$ & $X$ & - & $\mathrm{X}$ & - & $X$ & $X$ & $\mathrm{X}$ & - \\
\hline $4 \ldots$ & $\ldots \ldots$ & $X$ & $\mathrm{X}$ & - & $\mathrm{X}$ & $X$ & $\mathrm{X}$ & $\mathrm{X}$ & $\mathrm{X}$ & $X$ \\
\hline $5 \quad$. & $\ldots \ldots$ & $\mathrm{X}$ & $X$ & - & $\mathrm{X}$ & $\mathrm{X}$ & $\mathrm{X}$ & $\mathrm{X}$ & - & $\mathrm{X}$ \\
\hline $6 \ldots$ & $\ldots \ldots$ & $\mathrm{X}$ & $\mathrm{X}$ & $\mathrm{X}$ & - & - & - & $\mathrm{X}$ & - & - \\
\hline $7 \ldots$ & $\ldots \ldots$ & $X$ & $X$ & $\mathrm{X}$ & - & $X$ & $\mathrm{X}$ & $X$ & $X$ & - \\
\hline $8 \quad$. & $\ldots \ldots$ & $\mathrm{X}$ & $X$ & $\mathrm{X}$ & $\mathrm{X}$ & $X$ & - & $X$ & $\mathrm{X}$ & $\mathrm{X}$ \\
\hline $9 \quad$. & $\ldots \ldots$ & $X$ & $X$ & $\mathrm{X}$ & $\mathrm{X}$ & $X$ & $X$ & $X$ & $X$ & $X$ \\
\hline 10 . & $\ldots \ldots$ & $\mathrm{X}$ & $\mathrm{X}$ & - & - & $\mathrm{X}$ & $\mathrm{X}$ & $\mathrm{X}$ & - & $\mathrm{X}$ \\
\hline 11. & $\ldots \ldots$ & $\mathrm{X}$ & $\mathrm{X}$ & $\mathrm{X}$ & $\mathrm{X}$ & $X$ & $\mathrm{X}$ & $X$ & $X$ & $\mathrm{X}$ \\
\hline 12. & $\ldots \ldots$ & $X$ & $X$ & $\mathrm{X}$ & - & $X$ & - & $X$ & - & $X$ \\
\hline 13. & $\ldots \ldots$ & $\mathrm{X}$ & $X$ & $\mathrm{X}$ & $\mathrm{X}$ & $X$ & $\mathrm{X}$ & $X$ & $X$ & $X$ \\
\hline 14. & $\ldots \ldots$ & $\mathrm{X}$ & $\mathrm{X}$ & $\mathrm{X}$ & - & $X$ & $\mathrm{X}$ & $\mathrm{X}$ & - & - \\
\hline 15. & $\ldots \ldots$ & $X$ & $X$ & $X$ & $X$ & $X$ & $X$ & $X$ & $X$ & $X$ \\
\hline 16. & $\ldots \ldots$ & $X$ & $X$ & $X$ & $X$ & $X$ & - & $X$ & $\mathrm{X}$ & $X$ \\
\hline 17 . & $\ldots \ldots$ & $X$ & $X$ & $\mathrm{X}$ & $\mathrm{X}$ & $X$ & $X$ & $X$ & $\mathrm{X}$ & $X$ \\
\hline 18. & $\ldots \ldots$ & $X$ & $X$ & $X$ & $\mathrm{X}$ & $X$ & $X$ & $X$ & - & $X$ \\
\hline 19. & $\ldots \ldots$ & $X$ & $X$ & $X$ & $X$ & $X$ & $X$ & $X$ & - & $X$ \\
\hline 20. & $\ldots \ldots$ & $X$ & $X$ & $\mathrm{X}$ & $\mathrm{X}$ & - & $X$ & $X$ & $X$ & $X$ \\
\hline 21. & $\ldots \ldots$ & $X$ & $X$ & $X$ & $\mathrm{X}$ & - & - & $X$ & - & $X$ \\
\hline 22. & $\ldots \ldots$ & $X$ & $X$ & $X$ & $X$ & - & $X$ & $X$ & - & - \\
\hline 23. & $\ldots \ldots$ & $X$ & $X$ & $\mathrm{X}$ & $\mathrm{X}$ & $X$ & $X$ & $X$ & $X$ & $X$ \\
\hline 24. & $\ldots \ldots$ & $X$ & $X$ & $\mathrm{X}$ & $\mathrm{X}$ & $X$ & $X$ & $X$ & $\mathrm{X}$ & $X$ \\
\hline 25 & …... & $\mathrm{X}$ & $\mathrm{X}$ & $\mathrm{X}$ & $\mathrm{X}$ & $\mathrm{X}$ & $\mathrm{X}$ & $\mathrm{X}$ & $\mathrm{X}$ & $\mathrm{X}$ \\
\hline 26. & $\ldots \ldots$ & $X$ & $X$ & $\mathrm{X}$ & - & $X$ & - & $X$ & - & $X$ \\
\hline 27 . & $\ldots \ldots$ & $X$ & $X$ & $\mathrm{X}$ & $\mathrm{X}$ & $X$ & $X$ & $X$ & - & $X$ \\
\hline 28. & ....... & $X$ & $X$ & $\mathrm{X}$ & $\mathrm{X}$ & $X$ & $X$ & $X$ & - & $X$ \\
\hline 29. & $\ldots \ldots$ & $X$ & $X$ & $\mathrm{X}$ & - & - & - & $X$ & - & $X$ \\
\hline 30 & $\ldots \ldots$ & $X$ & $X$ & $\mathrm{X}$ & - & - & $X$ & $X$ & - & $X$ \\
\hline 31 & $\ldots \ldots$ & $X$ & $X$ & $\mathrm{X}$ & $X$ & $X$ & $X$ & $X$ & $X$ & $X$ \\
\hline 32 & $\ldots \ldots$ & $X$ & $X$ & $\mathrm{X}$ & $X$ & - & - & $X$ & $\mathrm{X}$ & $X$ \\
\hline 33 & $\ldots \ldots$ & $X$ & $X$ & $\mathrm{X}$ & $\mathrm{X}$ & - & $X$ & $X$ & $\mathrm{X}$ & $X$ \\
\hline 34 & $\ldots \ldots$ & $X$ & $X$ & $\mathrm{X}$ & $\mathrm{X}$ & - & $X$ & $X$ & $X$ & $X$ \\
\hline 35 & $\ldots \ldots$ & $X$ & $X$ & - & - & - & - & - & - & $\mathrm{X}$ \\
\hline 36 & $\ldots \ldots$ & $X$ & $X$ & $\mathrm{X}$ & $\mathrm{X}$ & $X$ & $X$ & $X$ & $\mathrm{X}$ & $X$ \\
\hline 37 & $\ldots \ldots$ & $X$ & $X$ & $X$ & $X$ & $X$ & $X$ & $X$ & $\mathrm{X}$ & $\mathrm{X}$ \\
\hline 38 & $\ldots \ldots$ & $X$ & $X$ & - & $X$ & $X$ & $X$ & $X$ & $\mathrm{X}$ & $\mathrm{X}$ \\
\hline 39 & $\ldots \ldots$ & $X$ & $X$ & $X$ & - & - & - & $X$ & - & $\mathrm{X}$ \\
\hline 40 & $\ldots \ldots$ & $X$ & $X$ & $\mathrm{X}$ & $\mathrm{X}$ & - & $X$ & $X$ & - & $X$ \\
\hline 41 & $\ldots \ldots$ & $X$ & $X$ & $X$ & $X$ & - & - & $X$ & - & - \\
\hline 42 & $\ldots \ldots$ & $X$ & $X$ & - & $X$ & - & $X$ & $X$ & $X$ & $X$ \\
\hline 43 & $\ldots \ldots$ & $X$ & $X$ & $X$ & - & - & - & $X$ & - & - \\
\hline 44 & $\ldots \ldots$ & $X$ & $X$ & $\mathrm{X}$ & - & - & $X$ & $X$ & - & $X$ \\
\hline 45 & $\ldots \ldots$ & $X$ & $X$ & $\mathrm{X}$ & - & $X$ & $X$ & $X$ & - & - \\
\hline 46 & …... & $\mathrm{X}$ & $\mathrm{X}$ & - & - & - & $\mathrm{X}$ & $\mathrm{X}$ & - & - \\
\hline 47 & $\ldots \ldots$ & $X$ & $X$ & $\mathrm{X}$ & $\mathrm{X}$ & - & $X$ & $X$ & - & $X$ \\
\hline 48 & $\ldots \ldots$ & $X$ & $X$ & $\mathrm{X}$ & $X$ & $X$ & $X$ & $X$ & $X$ & $\mathrm{X}$ \\
\hline 49 & $\ldots \ldots$ & $X$ & $X$ & $\mathrm{X}$ & - & - & - & $X$ & - & - \\
\hline 50 & $\ldots \ldots$ & $X$ & $X$ & $\mathrm{X}$ & $X$ & $X$ & $X$ & $X$ & $X$ & $\mathrm{X}$ \\
\hline 51 & $\ldots \ldots$ & $X$ & $X$ & - & $\mathrm{X}$ & $X$ & $X$ & $X$ & - & $\mathrm{X}$ \\
\hline 52 & $\ldots \ldots$ & $X$ & $X$ & $X$ & - & $X$ & $X$ & $X$ & - & $\mathrm{X}$ \\
\hline 53 & $\ldots \ldots$ & $X$ & $X$ & $\mathrm{X}$ & $\mathrm{X}$ & $X$ & $X$ & $X$ & $X$ & $\mathrm{X}$ \\
\hline 54 & $\ldots \ldots$ & $X$ & $X$ & $\mathrm{X}$ & $\mathrm{X}$ & $X$ & - & $X$ & $\mathrm{X}$ & $\mathrm{X}$ \\
\hline 55 & $\ldots \ldots$ & $X$ & $X$ & $\mathrm{X}$ & $\mathrm{X}$ & $X$ & $X$ & $\mathrm{X}$ & $X$ & $\mathrm{X}$ \\
\hline 56 & ...... & $X$ & $\mathrm{X}$ & $\mathrm{X}$ & $\mathrm{X}$ & $\mathrm{X}$ & $X$ & $\mathrm{X}$ & $\mathrm{X}$ & $\mathrm{X}$ \\
\hline 57 & $\ldots \ldots$ & $X$ & $X$ & $\mathrm{X}$ & $X$ & $X$ & $X$ & $X$ & $\mathrm{X}$ & $\mathrm{X}$ \\
\hline 58 & $\ldots \ldots$ & $X$ & $X$ & $X$ & $\mathrm{X}$ & $X$ & $X$ & $X$ & - & $\mathrm{X}$ \\
\hline 59 & $\ldots \ldots$ & $X$ & $X$ & $\mathrm{X}$ & $X$ & $X$ & $X$ & $X$ & $X$ & $\mathrm{X}$ \\
\hline
\end{tabular}


TABLE 4 (Continued)

\begin{tabular}{|c|c|c|c|c|c|c|c|c|c|c|}
\hline HRS & & 2MASS & SDSS & UV & $\mathrm{H} \alpha$ & IRAS & $20 \mathrm{~cm}$ & $\mathrm{HI}$ & $\mathrm{CO}$ & Spec \\
\hline 60 & $\ldots \ldots$ & $X$ & $X$ & $\mathrm{X}$ & $\mathrm{X}$ & $X$ & $X$ & $\mathrm{X}$ & $X$ & $\mathrm{X}$ \\
\hline 61 & $\ldots \ldots$ & $X$ & $X$ & $X$ & - & $X$ & - & $\mathrm{X}$ & - & $X$ \\
\hline 62 & $\ldots \ldots$ & $X$ & $X$ & $\mathrm{X}$ & $\mathrm{X}$ & - & $X$ & $\mathrm{X}$ & - & $X$ \\
\hline 63 & $\ldots \ldots$ & $X$ & $X$ & - & $\mathrm{X}$ & $X$ & - & $\mathrm{X}$ & $X$ & $X$ \\
\hline 64 & $\ldots \ldots$ & $X$ & $X$ & - & - & $X$ & - & $\mathrm{X}$ & - & $X$ \\
\hline 65 & $\ldots \ldots$ & $X$ & $X$ & $\mathrm{X}$ & - & $X$ & - & $\mathrm{X}$ & - & $X$ \\
\hline 66 & $\ldots \ldots$ & $X$ & $X$ & $X$ & $X$ & - & $X$ & $\mathrm{X}$ & $X$ & $X$ \\
\hline 67 & $\ldots \ldots$ & $X$ & $X$ & - & - & - & $X$ & $\mathrm{X}$ & - & $X$ \\
\hline 68 & $\ldots \ldots$ & $X$ & $X$ & $\mathrm{X}$ & - & - & $X$ & $\mathrm{X}$ & - & $X$ \\
\hline 69 & $\ldots \ldots$ & $X$ & $X$ & - & $X$ & $X$ & $X$ & $\mathrm{X}$ & $X$ & $X$ \\
\hline 70 & $\ldots \ldots$ & $X$ & - & $X$ & $X$ & $X$ & $X$ & $\mathrm{X}$ & - & $X$ \\
\hline 71 & $\ldots \ldots$ & $X$ & $X$ & $\mathrm{X}$ & - & $X$ & - & - & - & - \\
\hline 72 & $\ldots \ldots$ & $X$ & - & $X$ & - & $X$ & $X$ & $\mathrm{X}$ & - & $X$ \\
\hline 73 & $\ldots \ldots$ & $X$ & $X$ & - & $X$ & $X$ & - & $\mathrm{X}$ & $\mathrm{X}$ & $X$ \\
\hline 74 & $\ldots \ldots$ & $X$ & $X$ & - & $X$ & $X$ & $X$ & $\mathrm{X}$ & $X$ & $X$ \\
\hline 75 & $\ldots \ldots$ & $X$ & $X$ & - & - & - & - & $\mathrm{X}$ & - & $X$ \\
\hline 76 & $\ldots \ldots$ & $X$ & $X$ & - & $X$ & $X$ & - & $\mathrm{X}$ & - & $X$ \\
\hline 77 & $\ldots \ldots$ & $X$ & $X$ & $X$ & $X$ & $\mathrm{X}$ & $X$ & $\mathrm{X}$ & $X$ & $X$ \\
\hline 78 & $\ldots \ldots$ & $X$ & $X$ & $X$ & $X$ & $X$ & - & $\mathrm{X}$ & $X$ & $X$ \\
\hline 79 & $\ldots \ldots$ & $X$ & $X$ & X & $X$ & $X$ & $X$ & $\mathrm{X}$ & - & $X$ \\
\hline 80 & $\ldots \ldots$ & $X$ & $X$ & $X$ & $X$ & $\mathrm{X}$ & - & $\mathrm{X}$ & - & $X$ \\
\hline 81 & $\ldots \ldots$ & $X$ & $X$ & $X$ & $X$ & $X$ & $X$ & $\mathrm{X}$ & $X$ & $X$ \\
\hline 82 & $\ldots \ldots$ & $X$ & $X$ & $X$ & - & $X$ & $X$ & $\mathrm{X}$ & - & $X$ \\
\hline 83 & $\ldots \ldots$ & $X$ & $X$ & $X$ & - & $\mathrm{X}$ & - & $\mathrm{X}$ & - & $X$ \\
\hline 84 & $\ldots \ldots$ & $X$ & $X$ & $\mathrm{X}$ & $\mathrm{X}$ & $X$ & $X$ & $\mathrm{X}$ & $\mathrm{X}$ & $\mathrm{X}$ \\
\hline 85 & $\ldots \ldots$ & $X$ & $X$ & X & $X$ & $X$ & $X$ & $\mathrm{X}$ & $\mathrm{X}$ & $X$ \\
\hline 86 & $\ldots \ldots$ & $X$ & $X$ & $\mathrm{X}$ & $\mathrm{X}$ & $\mathrm{X}$ & $X$ & $\mathrm{X}$ & $X$ & $X$ \\
\hline 87 & $\ldots \ldots$ & $X$ & $X$ & $\mathrm{X}$ & - & $X$ & - & $\mathrm{X}$ & - & $X$ \\
\hline 88 & $\ldots \ldots$ & $X$ & $X$ & $\mathrm{X}$ & $X$ & $X$ & $X$ & $\mathrm{X}$ & $X$ & $X$ \\
\hline 89 & $\ldots \ldots$ & $X$ & $X$ & $\mathrm{X}$ & $\mathrm{X}$ & $X$ & $X$ & $\mathrm{X}$ & $X$ & $X$ \\
\hline 90 & $\ldots \ldots$ & $X$ & $\mathrm{X}$ & $\mathrm{X}$ & - & - & - & $\mathrm{X}$ & - & $X$ \\
\hline 91 & $\ldots \ldots$ & $X$ & $X$ & - & $\mathrm{X}$ & $X$ & $X$ & $\mathrm{X}$ & $X$ & $X$ \\
\hline 92 & $\ldots \ldots$ & $X$ & $X$ & - & $\mathrm{X}$ & $\mathrm{X}$ & - & $\mathrm{X}$ & - & $X$ \\
\hline 93 & $\ldots \ldots$ & $X$ & $X$ & $\mathrm{X}$ & - & $X$ & $X$ & $\mathrm{X}$ & $\mathrm{X}$ & - \\
\hline 94 & $\ldots \ldots$ & $X$ & $X$ & $X$ & $X$ & $X$ & - & $\mathrm{X}$ & $X$ & $X$ \\
\hline 95 & $\ldots \ldots$ & $X$ & $X$ & $\mathrm{X}$ & $X$ & $X$ & $X$ & $\mathrm{X}$ & $\mathrm{X}$ & $X$ \\
\hline 96 & $\ldots \ldots$ & $X$ & $X$ & - & $X$ & $X$ & $X$ & $\mathrm{X}$ & $\mathrm{X}$ & $X$ \\
\hline 97 & $\ldots \ldots$ & $X$ & $X$ & $\mathrm{X}$ & $\mathrm{X}$ & $X$ & $X$ & $\mathrm{X}$ & $\mathrm{X}$ & $\mathrm{X}$ \\
\hline 98 & $\ldots \ldots$ & $X$ & $X$ & $\mathrm{X}$ & $\mathrm{X}$ & $\mathrm{X}$ & $X$ & $\mathrm{X}$ & $\mathrm{X}$ & $X$ \\
\hline 99 & $\ldots \ldots$ & $X$ & $X$ & $\mathrm{X}$ & $X$ & $X$ & - & $\mathrm{X}$ & - & $\mathrm{X}$ \\
\hline 100 & $\ldots \ldots$ & $X$ & $X$ & $X$ & $X$ & $X$ & $X$ & $\mathrm{X}$ & $X$ & $X$ \\
\hline 101 & $\ldots$ & $X$ & $X$ & $\mathrm{X}$ & - & $X$ & - & $\mathrm{X}$ & $\mathrm{X}$ & - \\
\hline 102 & $\ldots$ & $X$ & $X$ & $\mathrm{X}$ & $\mathrm{X}$ & $X$ & $X$ & $\mathrm{X}$ & $\mathrm{X}$ & $X$ \\
\hline 103 & $\ldots \ldots$ & $X$ & $X$ & $X$ & $X$ & $X$ & - & $\mathrm{X}$ & $X$ & $X$ \\
\hline 104 & $\ldots$ & $X$ & $X$ & $\mathrm{X}$ & $\mathrm{X}$ & - & - & - & - & - \\
\hline 105 & $\ldots$ & $X$ & $X$ & - & - & - & - & $\mathrm{X}$ & - & $X$ \\
\hline 106 & $\ldots \ldots$ & $X$ & $X$ & $X$ & $X$ & $X$ & - & $\mathrm{X}$ & - & $X$ \\
\hline 107 & $\ldots$ & $X$ & $X$ & $\mathrm{X}$ & $X$ & - & - & $\mathrm{X}$ & - & $\mathrm{X}$ \\
\hline 108 & $\ldots$ & $X$ & $X$ & $\mathrm{X}$ & $X$ & - & - & $\mathrm{X}$ & - & $X$ \\
\hline 109 & $\ldots$ & $X$ & $X$ & $\mathrm{X}$ & $X$ & $X$ & $X$ & $\mathrm{X}$ & - & $X$ \\
\hline 110 & $\ldots$ & $X$ & $X$ & $\mathrm{X}$ & $X$ & $X$ & $X$ & $\mathrm{X}$ & $\mathrm{X}$ & $\mathrm{X}$ \\
\hline 111 & $\ldots$ & $X$ & $X$ & $\mathrm{X}$ & $\mathrm{X}$ & $X$ & $X$ & $\mathrm{X}$ & $\mathrm{X}$ & $X$ \\
\hline 112 & $\ldots$ & $X$ & $X$ & - & $X$ & $X$ & - & $\mathrm{X}$ & $X$ & $X$ \\
\hline 113 & $\ldots$ & $X$ & $X$ & $X$ & $X$ & $X$ & $X$ & $\mathrm{X}$ & $X$ & $X$ \\
\hline 114 & $\ldots$ & $X$ & $X$ & $\mathrm{X}$ & $X$ & $X$ & $X$ & $\mathrm{X}$ & $X$ & $X$ \\
\hline 115 & $\ldots$ & $X$ & $X$ & $\mathrm{X}$ & $X$ & - & - & $\mathrm{X}$ & - & $X$ \\
\hline 116 & $\ldots$ & $X$ & $X$ & - & $X$ & - & - & $\mathrm{X}$ & - & $\mathrm{X}$ \\
\hline 117 & $\ldots$ & $X$ & $X$ & - & $X$ & $X$ & $X$ & $\mathrm{X}$ & $X$ & $X$ \\
\hline 118 & $\ldots$ & $X$ & $X$ & $X$ & $X$ & $X$ & - & $\mathrm{X}$ & - & $X$ \\
\hline 119 & $\ldots$ & $X$ & $X$ & $X$ & $X$ & $X$ & $X$ & $\mathrm{X}$ & $X$ & $X$ \\
\hline
\end{tabular}


TABLE 4 (Continued)

\begin{tabular}{|c|c|c|c|c|c|c|c|c|c|c|}
\hline HRS & & 2MASS & SDSS & UV & $\mathrm{H} \alpha$ & IRAS & $20 \mathrm{~cm}$ & $\mathrm{HI}$ & $\mathrm{CO}$ & Spec \\
\hline 120 & $\ldots \ldots$ & $X$ & $X$ & $\mathrm{X}$ & $\mathrm{X}$ & $X$ & - & $\mathrm{X}$ & $\mathrm{X}$ & $\mathrm{X}$ \\
\hline 121 & $\ldots \ldots$ & $X$ & $\mathrm{X}$ & $\mathrm{X}$ & $\mathrm{X}$ & $X$ & $X$ & $\mathrm{X}$ & $\mathrm{X}$ & $X$ \\
\hline 122 & $\ldots \ldots$ & $X$ & $X$ & $\mathrm{X}$ & $X$ & $X$ & $\mathrm{X}$ & $\mathrm{X}$ & $\mathrm{X}$ & $\mathrm{X}$ \\
\hline 123 & $\ldots \ldots$ & $X$ & $X$ & X & $X$ & $X$ & - & $X$ & - & $X$ \\
\hline 124 & $\ldots \ldots$ & $X$ & $X$ & $\mathrm{X}$ & $\mathrm{X}$ & $X$ & $X$ & $\mathrm{X}$ & $\mathrm{X}$ & $X$ \\
\hline 125 & $\ldots \ldots$ & $\mathrm{X}$ & $\mathrm{X}$ & $\mathrm{X}$ & - & - & - & $\mathrm{X}$ & - & - \\
\hline 126 & $\ldots \ldots$ & $X$ & $\mathrm{X}$ & - & - & $X$ & - & $\mathrm{X}$ & - & - \\
\hline 127 & $\ldots \ldots$ & $X$ & $X$ & $\mathrm{X}$ & $\mathrm{X}$ & $X$ & $X$ & $\mathrm{X}$ & $\mathrm{X}$ & $\mathrm{X}$ \\
\hline 128 & $\ldots \ldots$ & $X$ & $X$ & $\mathrm{X}$ & $\mathrm{X}$ & $X$ & - & $\mathrm{X}$ & - & $X$ \\
\hline 129 & $\ldots \ldots$ & $X$ & $\mathrm{X}$ & $\mathrm{X}$ & - & $X$ & - & $\mathrm{X}$ & - & $X$ \\
\hline 130 & $\ldots \ldots$ & $X$ & $\mathrm{X}$ & $\mathrm{X}$ & $\mathrm{X}$ & $X$ & - & $\mathrm{X}$ & $\mathrm{X}$ & $X$ \\
\hline 131 & $\ldots \ldots$ & $X$ & $\mathrm{X}$ & $\mathrm{X}$ & $\mathrm{X}$ & - & - & $\mathrm{X}$ & - & $X$ \\
\hline 132 & $\ldots \ldots$ & $X$ & $X$ & $\mathrm{X}$ & $\mathrm{X}$ & $X$ & $X$ & $\mathrm{X}$ & - & $X$ \\
\hline 133 & $\ldots \ldots$ & $X$ & $X$ & $\mathrm{X}$ & $\mathrm{X}$ & $X$ & - & $\mathrm{X}$ & $X$ & $X$ \\
\hline 134 & $\ldots \ldots$ & $X$ & $\mathrm{X}$ & - & $\mathrm{X}$ & $X$ & - & $\mathrm{X}$ & - & $X$ \\
\hline 135 & $\ldots \ldots$ & $X$ & $X$ & $\mathrm{X}$ & $X$ & - & - & - & - & $X$ \\
\hline 136 & $\ldots \ldots$ & $X$ & $X$ & - & $X$ & $X$ & - & $X$ & $X$ & $X$ \\
\hline 137 & $\ldots .$. & $X$ & $X$ & $\mathrm{X}$ & - & - & - & $X$ & - & $X$ \\
\hline 138 & $\ldots \ldots$ & $X$ & $X$ & $\mathrm{X}$ & $X$ & $X$ & $X$ & $X$ & $X$ & - \\
\hline 139 & $\ldots \ldots$ & $X$ & $X$ & $\mathrm{X}$ & $X$ & $X$ & - & $X$ & - & $X$ \\
\hline 140 & $\ldots \ldots$ & $X$ & $X$ & $\mathrm{X}$ & $X$ & $X$ & - & $X$ & $X$ & $X$ \\
\hline 141 & $\ldots \ldots$ & $X$ & $X$ & $\mathrm{X}$ & $X$ & $X$ & - & $X$ & $\mathrm{X}$ & $X$ \\
\hline 142 & $\ldots \ldots$ & $X$ & $X$ & $\mathrm{X}$ & $X$ & $X$ & $X$ & $X$ & $X$ & $X$ \\
\hline 143 & $\ldots .$. & $X$ & $X$ & $\mathrm{X}$ & $X$ & $X$ & $X$ & $X$ & - & $X$ \\
\hline 144 & $\ldots \ldots$ & $X$ & $X$ & $\mathrm{X}$ & $X$ & $X$ & $X$ & $X$ & $\mathrm{X}$ & $X$ \\
\hline 145 & $\ldots \ldots$ & $X$ & $X$ & $\mathrm{X}$ & $\mathrm{X}$ & $X$ & - & $X$ & $X$ & $X$ \\
\hline 146 & $\ldots \ldots$ & $X$ & $X$ & $\mathrm{X}$ & $\mathrm{X}$ & $X$ & $X$ & $X$ & - & $X$ \\
\hline 147 & $\ldots \ldots$ & $X$ & $X$ & $\mathrm{X}$ & $X$ & $X$ & - & $X$ & - & $X$ \\
\hline 148 & $\ldots \ldots$ & $X$ & $X$ & $\mathrm{X}$ & $X$ & $X$ & $X$ & $X$ & $X$ & $X$ \\
\hline 149 & $\ldots \ldots$ & $X$ & $X$ & $\mathrm{X}$ & $X$ & $X$ & $X$ & $X$ & $\mathrm{X}$ & $X$ \\
\hline 150 & $\ldots \ldots$ & $X$ & $X$ & $\mathrm{X}$ & $X$ & $X$ & - & - & $\mathrm{X}$ & $X$ \\
\hline 151 & $\ldots \ldots$ & $X$ & $X$ & $\mathrm{X}$ & $X$ & $X$ & - & $X$ & $\mathrm{X}$ & $X$ \\
\hline 152 & $\ldots \ldots$ & $X$ & $X$ & $\mathrm{X}$ & $X$ & $X$ & $X$ & $X$ & $\mathrm{X}$ & $X$ \\
\hline 153 & $\ldots .$. & $X$ & $X$ & $\mathrm{X}$ & $X$ & $X$ & $X$ & $X$ & $\mathrm{X}$ & $X$ \\
\hline 154 & $\ldots \ldots$ & $X$ & $X$ & $\mathrm{X}$ & $X$ & $X$ & - & $X$ & $\mathrm{X}$ & $X$ \\
\hline 155 & $\ldots \ldots$ & $X$ & $X$ & $\mathrm{X}$ & - & - & - & $X$ & - & $\mathrm{X}$ \\
\hline 156 & $\ldots \ldots$ & $X$ & $X$ & $\mathrm{X}$ & $X$ & $X$ & $X$ & $X$ & $\mathrm{X}$ & $\mathrm{X}$ \\
\hline 157 & $\ldots \ldots$ & $X$ & $X$ & $\mathrm{X}$ & $X$ & $X$ & - & $X$ & $X$ & $\mathrm{X}$ \\
\hline 158 & $\ldots \ldots$ & $X$ & $X$ & $\mathrm{X}$ & $X$ & $X$ & $X$ & $\mathrm{X}$ & $X$ & $\mathrm{X}$ \\
\hline 159 & $\ldots \ldots$ & $X$ & $X$ & $\mathrm{X}$ & $X$ & $X$ & $X$ & $X$ & $X$ & $X$ \\
\hline 160 & $\ldots \ldots$ & $X$ & $X$ & $\mathrm{X}$ & $X$ & $X$ & $X$ & $X$ & $X$ & $X$ \\
\hline 161 & $\ldots \ldots$ & $X$ & $X$ & $\mathrm{X}$ & $X$ & $X$ & - & $X$ & $X$ & $X$ \\
\hline 162 & $\ldots \ldots$ & $X$ & $X$ & $\mathrm{X}$ & - & $X$ & - & $X$ & $\mathrm{X}$ & $X$ \\
\hline 163 & $\ldots \ldots$ & $X$ & $X$ & $\mathrm{X}$ & $X$ & $X$ & $X$ & $X$ & $\mathrm{X}$ & $\mathrm{X}$ \\
\hline 164 & $\ldots .$. & $X$ & $X$ & $\mathrm{X}$ & $\mathrm{X}$ & - & - & $X$ & $\mathrm{X}$ & $\mathrm{X}$ \\
\hline 165 & $\ldots \ldots$ & $X$ & $X$ & $\mathrm{X}$ & $\mathrm{X}$ & - & - & $X$ & - & $\mathrm{X}$ \\
\hline 166 & $\ldots \ldots$ & $X$ & $X$ & $\mathrm{X}$ & - & - & - & $X$ & - & $\mathrm{X}$ \\
\hline 167 & $\ldots \ldots$ & $X$ & $X$ & $\mathrm{X}$ & $X$ & $X$ & - & $X$ & - & $X$ \\
\hline 168 & $\ldots \ldots$ & $X$ & $X$ & X & X & $X$ & $X$ & $X$ & - & $\mathrm{X}$ \\
\hline 169 & $\ldots$. & $X$ & $X$ & $\mathrm{X}$ & $X$ & $X$ & - & $X$ & - & $\mathrm{X}$ \\
\hline 170 & $\ldots \ldots$ & $X$ & $X$ & $\mathrm{X}$ & $\mathrm{X}$ & $X$ & $X$ & $X$ & $X$ & $\mathrm{X}$ \\
\hline 171 & $\ldots \ldots$ & $X$ & $X$ & $\mathrm{X}$ & $\mathrm{X}$ & $X$ & $X$ & $X$ & $\mathrm{X}$ & $\mathrm{X}$ \\
\hline 172 & $\ldots \ldots$ & $X$ & $X$ & $\mathrm{X}$ & $\mathrm{X}$ & $X$ & $X$ & $X$ & $X$ & $X$ \\
\hline 173 & $\ldots \ldots$ & $X$ & $X$ & $\mathrm{X}$ & $\mathrm{X}$ & $X$ & $X$ & $X$ & $X$ & $X$ \\
\hline 174 & $\ldots \ldots$ & $X$ & $X$ & $\mathrm{X}$ & $X$ & $X$ & - & $\mathrm{X}$ & $\mathrm{X}$ & $\mathrm{X}$ \\
\hline 175 & $\ldots$. & $X$ & $X$ & $X$ & $\mathrm{X}$ & - & - & $X$ & - & $X$ \\
\hline 176 & $\ldots .$. & $X$ & $X$ & $\mathrm{X}$ & $\mathrm{X}$ & $X$ & - & $X$ & - & $\mathrm{X}$ \\
\hline 177 & $\ldots \ldots$ & $X$ & $X$ & $\mathrm{X}$ & $X$ & $X$ & $X$ & $X$ & $\mathrm{X}$ & $\mathrm{X}$ \\
\hline 178 & $\ldots \ldots$ & $X$ & $X$ & $\mathrm{X}$ & $X$ & - & $X$ & $X$ & $X$ & $X$ \\
\hline 179 & $\ldots \ldots$ & $X$ & $\mathrm{X}$ & $\mathrm{X}$ & $X$ & - & - & - & - & $\mathrm{X}$ \\
\hline
\end{tabular}


TABLE 4 (Continued)

\begin{tabular}{|c|c|c|c|c|c|c|c|c|c|c|}
\hline HRS & & 2MASS & SDSS & UV & $\mathrm{H} \alpha$ & IRAS & $20 \mathrm{~cm}$ & $\mathrm{HI}$ & $\mathrm{CO}$ & Spec \\
\hline 180 & $\ldots \ldots$ & $\mathrm{X}$ & $X$ & $\mathrm{X}$ & - & $X$ & - & $\mathrm{X}$ & - & $X$ \\
\hline 181 & $\ldots \ldots$ & $X$ & $X$ & $\mathrm{X}$ & - & - & - & - & - & - \\
\hline 182 & $\ldots \ldots$ & $X$ & $X$ & $X$ & $X$ & $X$ & $X$ & $\mathrm{X}$ & $X$ & $X$ \\
\hline 183 & $\ldots \ldots$ & $\mathrm{X}$ & $X$ & $X$ & $X$ & - & $X$ & - & $X$ & $X$ \\
\hline 184 & $\ldots \ldots$ & $X$ & $X$ & $X$ & $X$ & $X$ & - & $\mathrm{X}$ & $X$ & $X$ \\
\hline 185 & $\ldots \ldots$ & $X$ & $X$ & $X$ & $X$ & - & - & $\mathrm{X}$ & $X$ & $X$ \\
\hline 186 & $\ldots \ldots$ & $X$ & $X$ & - & - & - & - & $\mathrm{X}$ & $X$ & - \\
\hline 187 & $\ldots \ldots$ & $X$ & $X$ & $X$ & $X$ & $X$ & - & $\mathrm{X}$ & $X$ & $X$ \\
\hline 188 & $\ldots \ldots$ & $X$ & $\mathrm{X}$ & $X$ & $X$ & $X$ & $X$ & $\mathrm{X}$ & $X$ & $X$ \\
\hline 189 & $\ldots \ldots$ & $X$ & $X$ & $X$ & $X$ & $X$ & $X$ & $\mathrm{X}$ & - & $X$ \\
\hline 190 & $\ldots \ldots$ & $X$ & $X$ & $X$ & $X$ & $X$ & $X$ & $\mathrm{X}$ & $\mathrm{X}$ & $X$ \\
\hline 191 & $\ldots \ldots$ & $X$ & $\mathrm{X}$ & $X$ & $X$ & $X$ & - & $\mathrm{X}$ & - & $X$ \\
\hline 192 & $\ldots \ldots$ & $X$ & $X$ & $X$ & $X$ & $X$ & - & $\mathrm{X}$ & - & $X$ \\
\hline 193 & $\ldots \ldots$ & $X$ & $X$ & $\mathrm{X}$ & $X$ & $X$ & $X$ & $\mathrm{X}$ & $X$ & $X$ \\
\hline 194 & $\ldots$ & $X$ & $X$ & $X$ & $X$ & $X$ & - & $\mathrm{X}$ & $X$ & $X$ \\
\hline 195 & $\ldots \ldots$ & $X$ & $X$ & - & $X$ & - & - & $\mathrm{X}$ & $X$ & $X$ \\
\hline 196 & $\ldots \ldots$ & $X$ & $X$ & X & $X$ & $X$ & $X$ & $\mathrm{X}$ & $X$ & $X$ \\
\hline 197 & $\ldots \ldots$ & $X$ & $X$ & $X$ & $X$ & $X$ & $X$ & $\mathrm{X}$ & $X$ & $X$ \\
\hline 198 & $\ldots \ldots$ & $X$ & $X$ & $\mathrm{X}$ & $X$ & $X$ & - & $\mathrm{X}$ & $X$ & $X$ \\
\hline 199 & $\ldots \ldots$ & $X$ & $X$ & X & $X$ & $X$ & - & $\mathrm{X}$ & - & $X$ \\
\hline 200 & $\ldots \ldots$ & $X$ & $X$ & $\mathrm{X}$ & $X$ & $X$ & $X$ & $\mathrm{X}$ & $X$ & - \\
\hline 201 & $\ldots \ldots$ & $X$ & $X$ & $\mathrm{X}$ & $X$ & $X$ & $X$ & $\mathrm{X}$ & $X$ & $X$ \\
\hline 202 & $\ldots \ldots$ & $X$ & $X$ & - & - & - & - & - & - & $X$ \\
\hline 203 & $\ldots \ldots$ & $X$ & $X$ & $X$ & $X$ & $X$ & $X$ & $\mathrm{X}$ & $X$ & $X$ \\
\hline 204 & $\ldots \ldots$ & $X$ & $X$ & $\mathrm{X}$ & $X$ & $\mathrm{X}$ & $X$ & $\mathrm{X}$ & $X$ & $X$ \\
\hline 205 & $\ldots \ldots$ & $X$ & $X$ & X & $X$ & $X$ & $X$ & $\mathrm{X}$ & $X$ & $X$ \\
\hline 206 & $\ldots \ldots$ & $X$ & $X$ & $\mathrm{X}$ & $X$ & $\mathrm{X}$ & $X$ & $\mathrm{X}$ & $X$ & $X$ \\
\hline 207 & $\ldots \ldots$ & $X$ & $X$ & $\mathrm{X}$ & $X$ & $\mathrm{X}$ & $X$ & $\mathrm{X}$ & $X$ & $X$ \\
\hline 208 & $\ldots \ldots$ & $X$ & $X$ & X & $X$ & $X$ & - & $\mathrm{X}$ & $X$ & $X$ \\
\hline 209 & $\ldots \ldots$ & $X$ & - & $X$ & - & $X$ & $X$ & $\mathrm{X}$ & - & - \\
\hline 210 & $\ldots \ldots$ & $X$ & $X$ & $\mathrm{X}$ & - & - & - & $\mathrm{X}$ & - & $X$ \\
\hline 211 & $\ldots \ldots$ & $X$ & $X$ & $\mathrm{X}$ & $X$ & $X$ & $X$ & - & - & $X$ \\
\hline 212 & $\ldots \ldots$ & $X$ & $X$ & $\mathrm{X}$ & $X$ & $\mathrm{X}$ & $X$ & $\mathrm{X}$ & $X$ & $X$ \\
\hline 213 & $\ldots \ldots$ & $X$ & $X$ & $\mathrm{X}$ & $X$ & $\mathrm{X}$ & $X$ & $\mathrm{X}$ & $X$ & $X$ \\
\hline 214 & $\ldots \ldots$ & $X$ & $X$ & $\mathrm{X}$ & - & - & - & $\mathrm{X}$ & - & - \\
\hline 215 & $\ldots \ldots$ & $X$ & $X$ & $\mathrm{X}$ & $X$ & - & $X$ & X & $X$ & $X$ \\
\hline 216 & $\ldots \ldots$ & $X$ & $X$ & $\mathrm{X}$ & $X$ & $\mathrm{X}$ & $X$ & $\mathrm{X}$ & $X$ & $X$ \\
\hline 217 & $\ldots \ldots$ & $X$ & $X$ & $\mathrm{X}$ & $X$ & $X$ & $X$ & $\mathrm{X}$ & $X$ & $X$ \\
\hline 218 & $\ldots \ldots$ & $X$ & $X$ & $\mathrm{X}$ & - & - & - & X & - & - \\
\hline 219 & $\ldots \ldots$ & $X$ & $X$ & - & - & - & - & $\mathrm{X}$ & - & - \\
\hline 220 & $\ldots \ldots$ & $X$ & $X$ & $\mathrm{X}$ & $X$ & $\mathrm{X}$ & $\mathrm{X}$ & $\mathrm{X}$ & $X$ & $X$ \\
\hline 221 & $\ldots \ldots$ & $X$ & $X$ & $\mathrm{X}$ & $X$ & $X$ & - & $\mathrm{X}$ & $X$ & $X$ \\
\hline 222 & $\ldots \ldots$ & $X$ & $X$ & $X$ & $X$ & - & - & $\mathrm{X}$ & - & $X$ \\
\hline 223 & $\ldots \ldots$ & $X$ & $X$ & $\mathrm{X}$ & $X$ & - & - & $\mathrm{X}$ & - & $X$ \\
\hline 224 & $\ldots \ldots$ & $X$ & $X$ & $\mathrm{X}$ & $X$ & $X$ & - & $\mathrm{X}$ & $X$ & $X$ \\
\hline 225 & $\ldots \ldots$ & $X$ & $X$ & $\mathrm{X}$ & $X$ & - & - & $\mathrm{X}$ & - & $\mathrm{X}$ \\
\hline 226 & $\ldots \ldots$ & $X$ & $X$ & $X$ & $X$ & $X$ & - & $\mathrm{X}$ & - & $X$ \\
\hline 227 & $\ldots \ldots$ & $X$ & $X$ & $\mathrm{X}$ & $X$ & $X$ & $X$ & $\mathrm{X}$ & - & $\mathrm{X}$ \\
\hline 228 & $\ldots \ldots$ & $X$ & - & - & - & - & - & - & - & - \\
\hline 229 & $\ldots \ldots$ & $X$ & $X$ & $X$ & - & - & - & $\mathrm{X}$ & - & $\mathrm{X}$ \\
\hline 230 & $\ldots \ldots$ & $X$ & $X$ & $X$ & $X$ & $X$ & $X$ & $\mathrm{X}$ & $X$ & $X$ \\
\hline 231 & $\ldots \ldots$ & $X$ & $X$ & - & $X$ & $X$ & - & $\mathrm{X}$ & $X$ & $\mathrm{X}$ \\
\hline 232 & $\ldots \ldots$ & $X$ & $X$ & $\mathrm{X}$ & $X$ & $X$ & - & $\mathrm{X}$ & $X$ & $\mathrm{X}$ \\
\hline 233 & $\ldots$ & $X$ & $X$ & $X$ & $X$ & $\mathrm{X}$ & $X$ & $\mathrm{X}$ & $X$ & $\mathrm{X}$ \\
\hline 234 & $\ldots \ldots$ & $X$ & $X$ & - & - & - & $X$ & $\mathrm{X}$ & - & $\mathrm{X}$ \\
\hline 235 & $\ldots \ldots$ & $X$ & $X$ & $X$ & - & - & - & $\mathrm{X}$ & - & - \\
\hline 236 & $\ldots \ldots$ & $X$ & $X$ & $\mathrm{X}$ & - & - & - & - & - & $\mathrm{X}$ \\
\hline 237 & $\ldots \ldots$ & $X$ & $X$ & $\mathrm{X}$ & $X$ & $X$ & $X$ & $\mathrm{X}$ & $X$ & $\mathrm{X}$ \\
\hline 238 & $\ldots \ldots$ & $X$ & $X$ & - & - & - & - & $\mathrm{X}$ & - & $\mathrm{X}$ \\
\hline 239 & $\ldots \ldots$ & $X$ & $X$ & $\mathrm{X}$ & $X$ & $X$ & $X$ & $\mathrm{X}$ & $X$ & $X$ \\
\hline
\end{tabular}


TABLE 4 (Continued)

\begin{tabular}{|c|c|c|c|c|c|c|c|c|c|c|}
\hline HRS & & 2MASS & SDSS & UV & $\mathrm{H} \alpha$ & IRAS & $20 \mathrm{~cm}$ & $\mathrm{HI}$ & $\mathrm{CO}$ & Spec \\
\hline 240 & $\ldots \ldots$ & $\mathrm{X}$ & $\mathrm{X}$ & $X$ & - & - & - & $\mathrm{X}$ & - & $\bar{x}$ \\
\hline 241 & $\ldots \ldots$ & $\mathrm{X}$ & $\mathrm{X}$ & $X$ & $\mathrm{X}$ & - & $\mathrm{X}$ & $\mathrm{X}$ & $X$ & - \\
\hline 242 & $\ldots \ldots$ & $\mathrm{X}$ & $X$ & $\mathrm{X}$ & $\mathrm{X}$ & $X$ & $X$ & $\mathrm{X}$ & $X$ & $\mathrm{X}$ \\
\hline 243 & $\ldots \ldots$ & $\mathrm{X}$ & $\mathrm{X}$ & $\mathrm{X}$ & $\mathrm{X}$ & $X$ & - & $\mathrm{X}$ & - & - \\
\hline 244 & $\ldots \ldots$ & $\mathrm{X}$ & $X$ & $\mathrm{X}$ & $\mathrm{X}$ & $X$ & $\mathrm{X}$ & $\mathrm{X}$ & $X$ & $\mathrm{X}$ \\
\hline 245 & $\ldots \ldots$ & $\mathrm{X}$ & $X$ & $\mathrm{X}$ & $\mathrm{X}$ & $X$ & $\mathrm{X}$ & $\mathrm{X}$ & $X$ & $\mathrm{X}$ \\
\hline 246 & $\ldots \ldots$ & $\mathrm{X}$ & $\mathrm{X}$ & $\mathrm{X}$ & $\mathrm{X}$ & $\mathrm{X}$ & $\mathrm{X}$ & $\mathrm{X}$ & X & $\mathrm{X}$ \\
\hline 247 & $\ldots \ldots$ & $\mathrm{X}$ & $\mathrm{X}$ & $\mathrm{X}$ & $\mathrm{X}$ & $\mathrm{X}$ & $\mathrm{X}$ & $\mathrm{X}$ & $\mathrm{X}$ & $X$ \\
\hline 248 & $\ldots \ldots$ & $\mathrm{X}$ & $\mathrm{X}$ & $\mathrm{X}$ & - & - & - & - & - & $\mathrm{X}$ \\
\hline 249 & $\ldots$ & $\mathrm{X}$ & $\mathrm{X}$ & $\mathrm{X}$ & $\mathrm{X}$ & - & - & $\mathrm{X}$ & - & $\mathrm{X}$ \\
\hline 250 & $\ldots \ldots$ & $\mathrm{X}$ & $\mathrm{X}$ & $X$ & - & - & - & $\mathrm{X}$ & - & - \\
\hline 251 & $\ldots \ldots$ & $\mathrm{X}$ & $\mathrm{X}$ & $X$ & $\mathrm{X}$ & $X$ & $\mathrm{X}$ & $\mathrm{X}$ & $X$ & $\mathrm{X}$ \\
\hline 252 & $\ldots \ldots$ & $\mathrm{X}$ & $\mathrm{X}$ & $\mathrm{X}$ & - & $X$ & - & $\mathrm{X}$ & - & $\mathrm{X}$ \\
\hline 253 & $\ldots \ldots$ & $\mathrm{X}$ & $\mathrm{X}$ & $X$ & - & $X$ & $\mathrm{X}$ & $\mathrm{X}$ & - & - \\
\hline 254 & $\ldots \ldots$ & $X$ & $X$ & - & $\mathrm{X}$ & $X$ & $X$ & $X$ & $X$ & $\mathrm{X}$ \\
\hline 255 & $\ldots$ & $X$ & X & $X$ & $\mathrm{X}$ & $X$ & $X$ & $\mathrm{X}$ & X & $\mathrm{X}$ \\
\hline 256 & $\ldots \ldots$ & $X$ & $X$ & $X$ & - & $X$ & $X$ & $X$ & $X$ & - \\
\hline 257 & $\ldots .$. & $X$ & $X$ & $X$ & $\mathrm{X}$ & $X$ & - & $X$ & $X$ & $\mathrm{X}$ \\
\hline 258 & $\ldots$ & $X$ & $X$ & $X$ & $\mathrm{X}$ & $X$ & - & - & $X$ & - \\
\hline 259 & ...... & $X$ & $X$ & - & $\mathrm{X}$ & $X$ & $X$ & $X$ & $X$ & $\mathrm{X}$ \\
\hline 260 & $\ldots \ldots$ & $X$ & $X$ & $X$ & $\mathrm{X}$ & $X$ & $X$ & $X$ & $X$ & $\mathrm{X}$ \\
\hline 261 & $\ldots \ldots$ & $X$ & $X$ & $X$ & - & $X$ & - & $X$ & - & $\mathrm{X}$ \\
\hline 262 & $\ldots \ldots$ & $\mathrm{X}$ & $X$ & $X$ & $\mathrm{X}$ & $X$ & $X$ & $X$ & $X$ & $\mathrm{X}$ \\
\hline 263 & $\ldots .$. & $X$ & $X$ & $X$ & $\mathrm{X}$ & $X$ & $X$ & $X$ & $X$ & $X$ \\
\hline 264 & $\ldots \ldots$ & $\mathrm{X}$ & $X$ & $X$ & - & - & - & $\mathrm{X}$ & - & $\mathrm{X}$ \\
\hline 265 & ...... & $X$ & - & $\mathrm{X}$ & - & $X$ & $X$ & - & - & $\mathrm{X}$ \\
\hline 266 & $\ldots \ldots$ & $X$ & - & $X$ & $\mathrm{X}$ & $X$ & $X$ & $X$ & $X$ & $X$ \\
\hline 267 & $\ldots$ & $\mathrm{X}$ & $X$ & $\mathrm{X}$ & $\mathrm{X}$ & $X$ & $X$ & $X$ & - & $X$ \\
\hline 268 & $\ldots$. & $X$ & $X$ & $\mathrm{X}$ & $\mathrm{X}$ & $X$ & $X$ & $X$ & $X$ & $X$ \\
\hline 269 & $\ldots$. & $X$ & $X$ & $X$ & - & - & - & $X$ & - & $X$ \\
\hline 270 & $\ldots$ & $\mathrm{X}$ & $X$ & $\mathrm{X}$ & - & $\mathrm{X}$ & - & $\mathrm{X}$ & $X$ & $\mathrm{X}$ \\
\hline 271 & $\ldots \ldots$ & $\mathrm{X}$ & $X$ & $\mathrm{X}$ & $\mathrm{X}$ & $X$ & $X$ & $X$ & - & $\mathrm{X}$ \\
\hline 272 & $\ldots$ & $X$ & $X$ & $X$ & - & - & - & $X$ & - & $X$ \\
\hline 273 & $\ldots .$. & $X$ & $X$ & $X$ & $\mathrm{X}$ & $X$ & - & $X$ & $\mathrm{X}$ & $\mathrm{X}$ \\
\hline 274 & $\ldots \ldots$ & $\mathrm{X}$ & $X$ & $\mathrm{X}$ & $\mathrm{X}$ & $X$ & - & $X$ & $\mathrm{X}$ & $\mathrm{X}$ \\
\hline 275 & $\ldots$ & $\mathrm{X}$ & - & $\mathrm{X}$ & $\mathrm{X}$ & $X$ & $\mathrm{X}$ & $\mathrm{X}$ & $\mathrm{X}$ & $\mathrm{X}$ \\
\hline 276 & $\cdots$ & $X$ & $X$ & $\mathrm{X}$ & $\mathrm{X}$ & $X$ & $X$ & $\mathrm{X}$ & $\mathrm{X}$ & $\mathrm{X}$ \\
\hline 277 & $\ldots \ldots$ & $X$ & $X$ & $\mathrm{X}$ & - & - & - & $X$ & - & $\mathrm{X}$ \\
\hline 278 & $\ldots .$. & $X$ & $X$ & - & $\mathrm{X}$ & - & - & $X$ & - & $\mathrm{X}$ \\
\hline 279 & $\ldots$ & $X$ & $X$ & $\mathrm{X}$ & $\mathrm{X}$ & $X$ & - & $X$ & - & $\mathrm{X}$ \\
\hline 280 & ...... & $X$ & $X$ & $\mathrm{X}$ & $\mathrm{X}$ & $X$ & $X$ & $X$ & $X$ & $\mathrm{X}$ \\
\hline 281 & $\ldots \ldots$ & $X$ & $X$ & $\mathrm{X}$ & $\mathrm{X}$ & - & - & $X$ & - & $\mathrm{X}$ \\
\hline 282 & $\ldots \ldots$ & $\mathrm{X}$ & $X$ & - & - & - & - & - & - & $X$ \\
\hline 283 & $\ldots$. & $X$ & $X$ & $\mathrm{X}$ & $\mathrm{X}$ & $X$ & $\mathrm{X}$ & $X$ & $X$ & $X$ \\
\hline 284 & $\ldots \ldots$ & $X$ & - & $\mathrm{X}$ & - & $X$ & $X$ & $X$ & X & $\mathrm{X}$ \\
\hline 285 & $\ldots \ldots$ & $X$ & $X$ & $\mathrm{X}$ & $\mathrm{X}$ & $X$ & $X$ & $X$ & X & $\mathrm{X}$ \\
\hline 286 & $\ldots$ & $\mathrm{X}$ & $\mathrm{X}$ & $\mathrm{X}$ & $\mathrm{X}$ & - & - & $\mathrm{X}$ & $\mathrm{X}$ & $\mathrm{X}$ \\
\hline 287 & $\ldots \ldots$ & $X$ & $X$ & $\mathrm{X}$ & $\mathrm{X}$ & $X$ & $X$ & $X$ & $X$ & $\mathrm{X}$ \\
\hline 288 & $\ldots \ldots$ & $\mathrm{X}$ & - & $X$ & $\mathrm{X}$ & $X$ & $X$ & $X$ & $X$ & $X$ \\
\hline 289 & $\ldots .$. & $X$ & - & $X$ & $\mathrm{X}$ & $X$ & $X$ & $X$ & $X$ & $X$ \\
\hline 290 & $\ldots \ldots$ & $\mathrm{X}$ & $X$ & $\mathrm{X}$ & $\mathrm{X}$ & $X$ & $X$ & $X$ & - & $\mathrm{X}$ \\
\hline 291 & $\ldots \ldots$ & $X$ & $X$ & $\mathrm{X}$ & - & - & - & $X$ & $\mathrm{X}$ & - \\
\hline 292 & $\ldots \ldots$ & $X$ & $X$ & $X$ & $\mathrm{X}$ & $X$ & $X$ & $X$ & $\mathrm{X}$ & $X$ \\
\hline 293 & $\ldots \ldots$ & $\mathrm{X}$ & $X$ & $X$ & $\mathrm{X}$ & $X$ & $X$ & $X$ & $X$ & $X$ \\
\hline 294 & $\ldots .$. & $X$ & $X$ & - & $\mathrm{X}$ & $X$ & $X$ & $X$ & - & $\mathrm{X}$ \\
\hline 295 & $\ldots \ldots$ & $X$ & $X$ & $\mathrm{X}$ & $\mathrm{X}$ & $X$ & $X$ & $X$ & $X$ & $\mathrm{X}$ \\
\hline 296 & $\ldots$ & $\mathrm{X}$ & $X$ & $\mathrm{X}$ & $\mathrm{X}$ & $\mathrm{X}$ & $X$ & $\mathrm{X}$ & - & - \\
\hline 297 & $\ldots \ldots$ & $X$ & $X$ & $\mathrm{X}$ & $\mathrm{X}$ & $X$ & $X$ & $X$ & $X$ & $X$ \\
\hline 298 & $\ldots \ldots$ & $X$ & $X$ & $\mathrm{X}$ & $\mathrm{X}$ & $X$ & $X$ & $X$ & - & $X$ \\
\hline 299 & $\ldots .$. & $X$ & $X$ & $X$ & $\mathrm{X}$ & $X$ & - & $X$ & $X$ & $\mathrm{X}$ \\
\hline
\end{tabular}


TABLE 4 (Continued)

\begin{tabular}{|c|c|c|c|c|c|c|c|c|c|c|}
\hline HRS & & 2MASS & SDSS & UV & $\mathrm{H} \alpha$ & IRAS & $20 \mathrm{~cm}$ & $\mathrm{HI}$ & $\mathrm{CO}$ & Spec \\
\hline 300 & $\ldots .$. & $X$ & X & - & X & $X$ & - & $\mathrm{X}$ & - & X \\
\hline 301 & $\ldots .$. & $X$ & X & X & X & X & - & $\mathrm{X}$ & X & X \\
\hline 302 & $\ldots \ldots$ & X & X & $X$ & X & $X$ & - & $\mathrm{X}$ & - & X \\
\hline 303 & $\ldots .$. & X & X & $\mathrm{X}$ & X & $X$ & X & - & - & X \\
\hline 304 & $\ldots .$. & $X$ & X & $\mathrm{X}$ & X & $X$ & - & $\mathrm{X}$ & $\mathrm{X}$ & X \\
\hline 305 & $\ldots \ldots$ & X & X & $X$ & X & - & - & $\mathrm{X}$ & - & X \\
\hline 306 & $\ldots .$. & $X$ & $X$ & $X$ & - & $X$ & $X$ & $\mathrm{X}$ & X & X \\
\hline 307 & $\ldots .$. & X & X & $X$ & X & $X$ & X & $\mathrm{X}$ & $\mathrm{X}$ & X \\
\hline 308 & $\ldots \ldots$ & X & X & X & X & - & - & - & - & X \\
\hline 309 & $\ldots$. & X & $X$ & - & X & $X$ & - & $\mathrm{X}$ & - & X \\
\hline 310 & $\ldots \ldots$ & X & X & X & X & - & X & $\mathrm{X}$ & $X$ & X \\
\hline 311 & $\ldots \ldots$ & X & X & $X$ & X & $X$ & X & $\mathrm{X}$ & $\mathrm{X}$ & X \\
\hline 312 & $\ldots .$. & $X$ & $X$ & $X$ & - & $X$ & - & $\mathrm{X}$ & - & - \\
\hline 313 & $\ldots \ldots$ & X & X & X & X & $X$ & X & $\mathrm{X}$ & X & X \\
\hline 314 & $\ldots \ldots$ & X & X & $X$ & X & $X$ & X & $\mathrm{X}$ & - & X \\
\hline 315 & $\ldots$. & X & $X$ & $\mathrm{X}$ & X & - & - & $\mathrm{X}$ & - & X \\
\hline 316 & $\ldots \ldots$ & X & X & $\mathrm{X}$ & - & - & - & $\mathrm{X}$ & - & - \\
\hline 317 & $\ldots$. & $X$ & X & X & - & - & - & $\mathrm{X}$ & - & $X$ \\
\hline 318 & $\ldots$. & X & $X$ & $\mathrm{X}$ & X & $X$ & $X$ & $\mathrm{X}$ & $\mathrm{X}$ & X \\
\hline 319 & $\ldots \ldots$ & X & X & X & X & $X$ & X & $\mathrm{X}$ & $\mathrm{X}$ & X \\
\hline 320 & $\ldots .$. & $X$ & X & $X$ & X & $X$ & X & $\mathrm{X}$ & X & $X$ \\
\hline 321 & $\ldots$. & $\mathrm{X}$ & $X$ & $\mathrm{X}$ & - & $X$ & X & $\mathrm{X}$ & - & X \\
\hline 322 & $\ldots \ldots$ & $X$ & X & $\mathrm{X}$ & X & $X$ & - & $\mathrm{X}$ & - & $\mathrm{X}$ \\
\hline 323 & $\ldots$. & $X$ & X & X & X & $X$ & X & $\mathrm{X}$ & $X$ & $X$ \\
\hline
\end{tabular}

Centre since some members are also part of our consortium. This ensures we have access to the latest calibration files and bug fixes. Aside from the default data processing, the pipeline will also include new steps developed by the key-project teams (such as cosmic ray removal and baseline subtraction) for producing the most accurate representation of large-scale structures, such as the extended emission around galaxies. Additional postprocessing steps will be applied to prepare the data so that it can be easily used by the broader astronomical community. This will include reformatting the headers of the fits files so that they are easy to understand. Furthermore, the data will have been visually inspected so as to ensure that they suffer from no severe artefacts related to the observations or data reduction, and in cases where problems are identified, the data will be manually reprocessed to obtain the best results. After the proprietary time the team plans to make the reduced data available to the community through a dedicated web page. Herschel and ancillary data will be archived in an Information System at the Laboratoire d'Astrophysique de Marseille developped under the SITools middleware interface (http://vds.cnes .fr/sitools/) with a Postgresql database. Images will be stored as fits files, and distributed in fits format while catalogs will be distributed in VO table and ASCII (cvs) format. The SPIRE data delivered by the team will be optimized for use by the wider astronomical community. Given the legacy nature of this project, we will also present through an interactive database all the ancillary data, X, UV, optical, near- and far-IR, submillimeter, and radio continuum fluxes and images, optical and line spectroscopy and derived parameters (structural parameters, metallicities, SFR...) with the aim of providing the community with a unique data set, a suitable reference for future studies.

\section{THE STATISTICAL PROPERTIES OF THE HERSCHEL REFERENCE SAMPLE}

In this section, we investigate how representative the HRS is of the galaxy population as a whole, thus unbiased versus cosmic variance. This is necessary since, as selected, the HRS is biased versus galaxies located in a high density environment (Virgo). One way to test this is to see whether the luminosity distributions at different wavelengths in the HRS are similar to the local galaxy luminosity functions at the corresponding frequencies.

Shown in Figure 3 is a comparison of the HRS luminosity distribution in the $K$ band (histogram) to the $K$-band luminosity function of Cole et al. (2001) (dotted-dashed line; $\left.\alpha=0.96 \pm 0.05, M_{K}^{*}=-23.44 \pm 0.03\right)$ and Kochanek et al. (2001) (solid line; $\alpha=1.09 \pm 0.06, M_{K}^{*}=-23.39 \pm 0.05$ ), this last also considering separately early- (red dotted line; $\alpha=0.92 \pm 0.10, M_{K}^{*}=-23.53 \pm 0.06$; color details can be seen in the online Figures) and late-type (blue dashed line; $\left.\alpha=0.87 \pm 0.09, M_{K}^{*}=-22.98 \pm 0.06\right)$ galaxies. The Kochanek et al. (2001) values of the $K$-band luminosity function have 


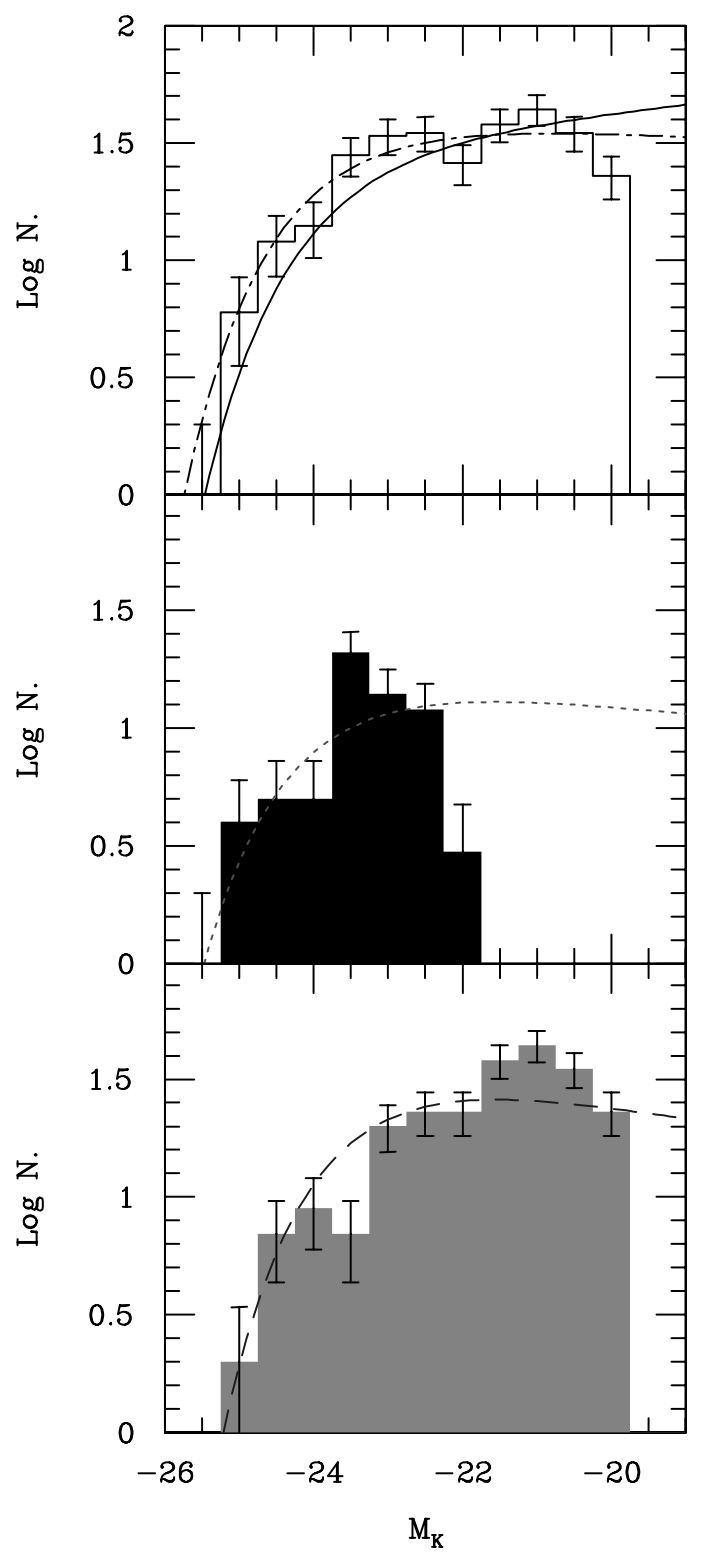

FIG. 3. $-K$ band luminosity distribution of the HRS for all galaxies is compared to the 2MASS $K$ band luminosity function of Kochanek et al. (2001) (solid line) and Cole et al. (2001) (dotted-dashed line), both in upper panel. The $K$ band luminosity distributions of the E-SO-S0a (center panel) and SaSd-Im-BCD (lower panel) galaxies in the HRS are compared to the Kochanek et al. (2001) $K$ band luminosity function of early-type (center panel, dotted line) and late-type (lower panel,dashed line) galaxies. Poisson errors are also indicated. See the electronic edition of the PASP for a color version of this figure.

been corrected to take into account the different assumed $\mathrm{H}_{0}$, while the Cole et al. (2001) $M_{K S}^{*}$ Kron magnitudes have been converted into total magnitudes as in this work. The zero point of the plotted luminosity functions is determined assuming the same number of objects as HRS within the given magnitude limit. Figure 3 shows that, when considered from the perspective of the $K$ band, the HRS is a good approximation to a volume-limited sample down to an absolute magnitude of

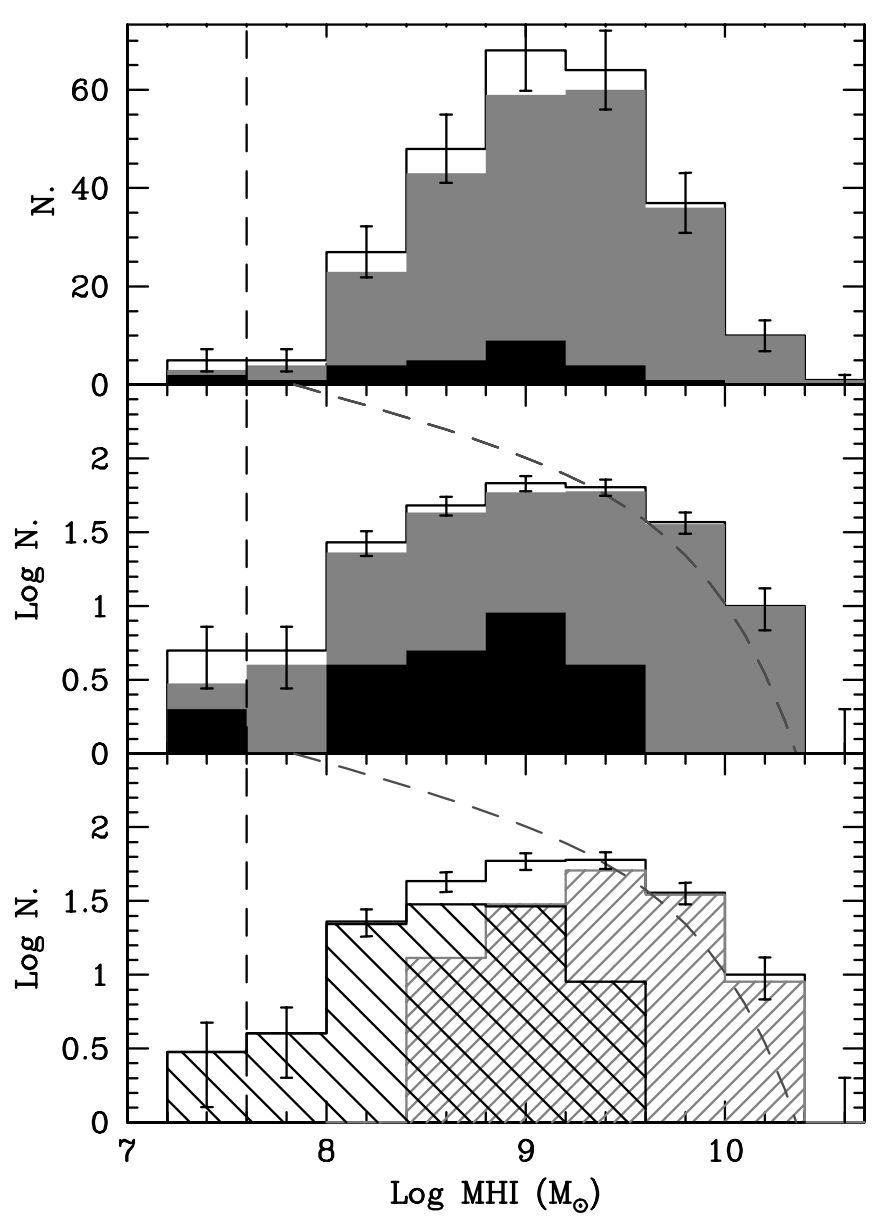

FIG. 4.-Atomic hydrogen mass distribution in linear (upper panel) and logarithmic (middle panel) scales for the HI-detected HRS galaxies (empty histogram). The black and gray histograms are for early-type (E-S0-S0a, 35\% detected) and late-type (Sa-Sd-Im-BCD; 93\% detected) galaxies, respectively. Lower panel: Atomic hydrogen mass distribution for late-type galaxies with a normal HI gas content (HI-deficiency $<0.4$; tiny spaced hashed histogram) and gas-poor galaxies (HI-deficiency $\geq 0.4$; large spaced hashed histogram). Curved dashed line is the $\mathrm{HI}$ mass function determined by Zwaan et al. (2005); vertical dashed line indicates the detection limit of the ALFALFA survey $(2.5 \mathrm{mJy})$ at the distance of $20 \mathrm{Mpc}$. See the electronic edition of the PASP for a color version of this figure.

$\mathbf{M}_{K} \simeq-20$. This absolute magnitude limit is relatively high and explains why the HRS is underrepresented in Im and BCD galaxies (Fig. 2). The sample includes only the brightest ellipticals and lenticulars, whose $K$-band luminosity function ends at $M_{K} \sim-18$, while it does not include any quiescent dwarf system $\left(\mathrm{M}_{K} \geq-20\right)$.

Figure 4 shows the HI mass distribution of the HI-detected HRS early-type (black histogram) and late-type (gray histogram) galaxies compared to the HIPASS HI mass function determined by Zwaan et al. (2005) (dashed line, $\alpha=-1.37 \pm 0.03, \mathrm{M}_{\mathrm{HI}}^{*}=10^{9.8 \pm 0.03} M_{\odot}$ and $\phi^{*}$ arbitrarily normalized to match the data). The sample includes galaxies with $\mathrm{HI}$ masses ranging between $\sim 10^{8}$ and $10^{10} M_{\odot}$ but it is 
clearly under-representing galaxies with $\mathrm{HI}$ masses in the range $10^{7.5} \mathrm{M}_{\odot} \leq \mathrm{M}_{\mathrm{HI}} \leq 10^{9} M_{\odot}$ which are easily detectable by an $\mathrm{HI}$ blind survey such as ALFALFA. The HIPASS HI mass function is dominated by gas rich, star-forming galaxies, while the HRS also includes quiescent, gas-poor early-types and HIdeficient cluster galaxies, as clearly observed in the Virgo cluster (Gavazzi et al. 2005; 2008) or in A1367 (Cortese et al. 2008b). The difference between the HIPASS mass function and the HRS HI mass distribution is due to (i) the fact that the HRS is $K$ band selected and thus does not include gas rich, low-luminosity star-forming systems with $\mathrm{HI}$ masses in the range $10^{7.5} \leq \mathrm{MHI} \leq 10^{8.5} \mathrm{M}_{\odot}$ with $K$ band magnitudes $12 \leq K_{\text {Stot }} \leq 16$, and (ii) the presence of cluster HI-deficient late-type galaxies. Indeed, the HI mass distribution of galaxies with a normal HI gas content $\left(\mathrm{HI}-\right.$ deficiency $\left.{ }^{31}<0.4\right)$ is more similar to the HIPASS mass function than the distribution of gas-poor objects (HI-deficiency $\geq 0.4$ ). Most of the late-type HI-deficient objects (HI-deficiency $\geq 0.4$ ) in the HRS are indeed located in the core of the Virgo cluster as shown in Figure 5. Galaxies in the outskirt of the Virgo cluster or in the surrounding clouds have a normal HI gas content (see Table 5) and can be generally considered as unperturbed objects (unless belonging to groups or pairs) in the study of the effects of the environment on the dust properties of galaxies. Indeed, despite the relatively poor statistics, the average HI-deficiency of late-type galaxies in the Coma I Cloud, Leo Cloud, Ursa Major Cloud and Southern Spur, Crater Cloud, Canes Venatici Spur, Canes VenaticiCamelopardalis Cloud, and Virgo-Libra Cloud is consistent with that of unperturbed galaxies in the reference sample of Haynes \& Giovanelli (1984; HI-deficiency $\leq 0.3$ ). The HIdeficiency of late-type galaxies in the different substructures of the Virgo cluster are generally consistent with those observed in a larger sample by Gavazzi et al. (1999a). The only exception is the Virgo E Cloud that we found to be dominated by HIdeficient objects. We notice, however, that the majority of the most HI-deficient galaxies (HI-deficiency $>1.0$ ) of the HRS belonging to this substructure are classified as early types in the VCC. The Coma I Cloud, previously thought to include gas-deficient objects (Garcia-Barreto et al. 1994; Gerin \& Casoli 1994), is composed of galaxies with a normal gas content (Boselli \& Gavazzi 2009). A detailed and complete study of the HI properties of late-type galaxies belonging to the other Clouds has not been done so far. A rather normal HI gas content of the spiral galaxies in the Ursa Major Cluster, a substructure of the

\footnotetext{
${ }^{31}$ The HI-deficiency parameter is defined as as the logarithmic difference between the average $\mathrm{HI}$ mass of a reference sample of isolated galaxies of similar type and linear dimension and the HI mass actually observed in individual objects: $\mathrm{HI}-\operatorname{def}=\log \mathrm{MHI}_{\text {ref }}-\log \mathrm{MHI}_{\text {obs }}$. According to Haynes \& Giovanelli (1984), $\log \mathrm{MHI}_{\mathrm{ref}}=a+b \times \log (\operatorname{diam})$, where $a$ and $b$ are weak functions of the Hubble type and diam is the linear diameter of the galaxy (see Gavazzi et al. 2005).
}

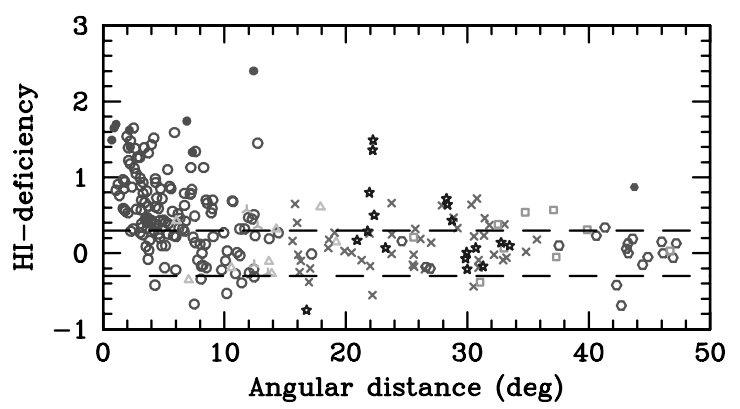

FIG. 5.-Variation of the HI-deficiency parameter as a function of the angular distance from the core of the Virgo cluster (M87). Empty symbols are for HIdetected galaxies, filled symbols for upper limits. See the electronic edition of the PASP for a color version of this figure with key to symbols.

Ursa Major Cloud, has been observed by Verheijen \& Sancisi (2001).

Figure 6 shows the far-IR luminosity distribution of the HRS early-type (black histogram) and late-type (gray histogram) galaxies detected by IRAS at $60 \mu \mathrm{m}$ compared the IRAS $60 \mu \mathrm{m}$ luminosity function determined by Takeuchi et al. (2003) $\left(\alpha=1.23 \pm 0.04, L_{60}^{*}=8.85 \times 10^{8} L_{\odot}\right.$, and $\phi^{*}$ in arbitrary units). The luminosity distribution of the HRS follows the luminosity function over 2 orders of magnitude. The lowluminosity cutoff is due to the detection limit of the IRAS survey. The lack of very luminous galaxies is because Luminous (LIRGs, $L_{60 \mu \mathrm{m}}=10^{11} L_{\odot}$ ) and Ultra Luminous (ULIRGs, $\left.L_{60 \mu \mathrm{m}}=10^{12} L_{\odot}\right)$ Infrared Galaxies are quite rare in the nearby universe and in particular inside rich clusters (Boselli \& Gavazzi 2006).

TABLE 5

The Average Hi Deficiency of Late-Type Galaxies IN THE SUBSTRUCTURES

\begin{tabular}{|c|c|c|c|}
\hline Substructure & Average & $\begin{array}{l}\text { Standard } \\
\text { Deviation }\end{array}$ & $\begin{array}{c}\text { Number of } \\
\text { Objects }\end{array}$ \\
\hline Virgo A & 0.91 & \pm 0.47 & 33 \\
\hline Virgo B & 0.71 & \pm 0.46 & 25 \\
\hline Virgo N Cloud ... & 0.39 & \pm 0.24 & 12 \\
\hline Virgo E Cloud $\ldots \ldots \ldots \ldots \ldots$ & 0.83 & \pm 0.53 & 12 \\
\hline Virgo $\mathrm{S}$ Cloud $\quad \ldots \ldots \ldots \ldots \ldots$ & 0.47 & \pm 0.47 & 26 \\
\hline Virgo Outskirts $\ldots \ldots \ldots \ldots \ldots$ & 0.26 & \pm 0.60 & 38 \\
\hline Coma I Cloud .... & 0.07 & \pm 0.35 & 8 \\
\hline Leo Cloud $\ldots \ldots \ldots \ldots \ldots \ldots$ & 0.11 & \pm 0.31 & 45 \\
\hline Ursa Major Cloud $\quad \ldots \ldots \ldots \ldots$ & 0.05 & \pm 0.34 & 15 \\
\hline $\begin{array}{l}\text { Ursa Major Southern } \\
\text { Spur } \quad \ldots \ldots \ldots \ldots \ldots\end{array}$ & -0.02 & \pm 0.20 & 4 \\
\hline Crater Cloud $\quad \ldots \ldots \ldots \ldots \ldots$ & 0.13 & \pm 0.40 & 4 \\
\hline $\begin{array}{l}\text { Canes Ven. Spur \& } \\
\text { Camelopardalis } \quad \ldots . \ldots \ldots \ldots\end{array}$ & 0.19 & \pm 0.03 & 9 \\
\hline Virgo-Libra Cloud $\ldots \ldots \ldots \ldots$ & 0.31 & \pm 0.35 & 18 \\
\hline
\end{tabular}

NoTE.-Upper limits are considered as detections. 


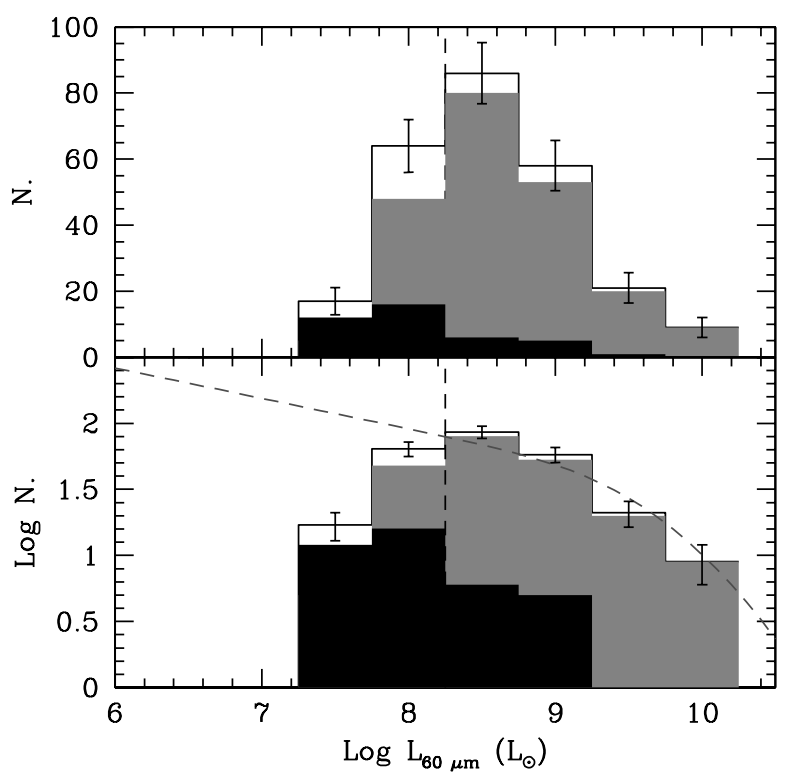

FIG. 6.-Infrared luminosity distribution in linear (upper panel) and logarithmic (lower panel) scales for the FIR $(60 \mu \mathrm{m})$ detected HRS galaxies (empty histogram). The black and gray histograms are for early-type (E-S0-SOa) and late-type (Sa-Sd-Im-BCD) galaxies respectively. Curved dashed line is the IRAS $60 \mu \mathrm{m}$ luminosity function determined by Takeuchi et al. (2003); vertical dashed line indicates the typical detection limit of IRAS $(0.4 \mathrm{mJy})$ at the distance of $20 \mathrm{Mpc}$. See the electronic edition of the PASP for a color version of this figure.

Figure 7 shows the radio continuum $(1.4 \mathrm{GHz})$ luminosity distribution of the radio-detected HRS early-type (black histogram) and late-type (gray histogram) galaxies, as compared to the $2 \mathrm{dF} /$ NVSS luminosity function of Mauch \& Sadler (2007) (solid line). This radio luminosity function includes the contribution from both AGN (dashed line) and quiescent (dotted line) galaxies. The radio luminosity distribution of the HRS is quite typical of normal, nearby galaxies $\left(10^{20} \leq L_{\text {Radio }} \leq\right.$ $10^{22} \mathrm{~W} \mathrm{~Hz}^{-1}$; Condon et al. 2002). The two brightest sources are the radio galaxies M87 (Virgo A, $L_{\text {Radio }}=10^{24.8} \mathrm{~W} \mathrm{~Hz}^{-1}$ ) and M84 $\left(L_{\text {Radio }}=10^{23.3} \mathrm{~W} \mathrm{~Hz}^{-1}\right)$. The radio-continuum luminosity distribution as a whole agrees well with the field luminosity function, indicating that the contamination of cluster galaxies with an enhanced radio-continuum emission is minimal (Gavazzi \& Boselli 1999c, 1999d).

In summary, since the $K$-band light traces stellar mass, the good agreement between the HRS luminosity distribution and $K$-band luminosity function implies that the HRS can be treated as a volume-limited sample down to a minimum stellar mass. Figure 6 shows that the HRS is also representative of a farIR selected sample once the most active and rare in the local

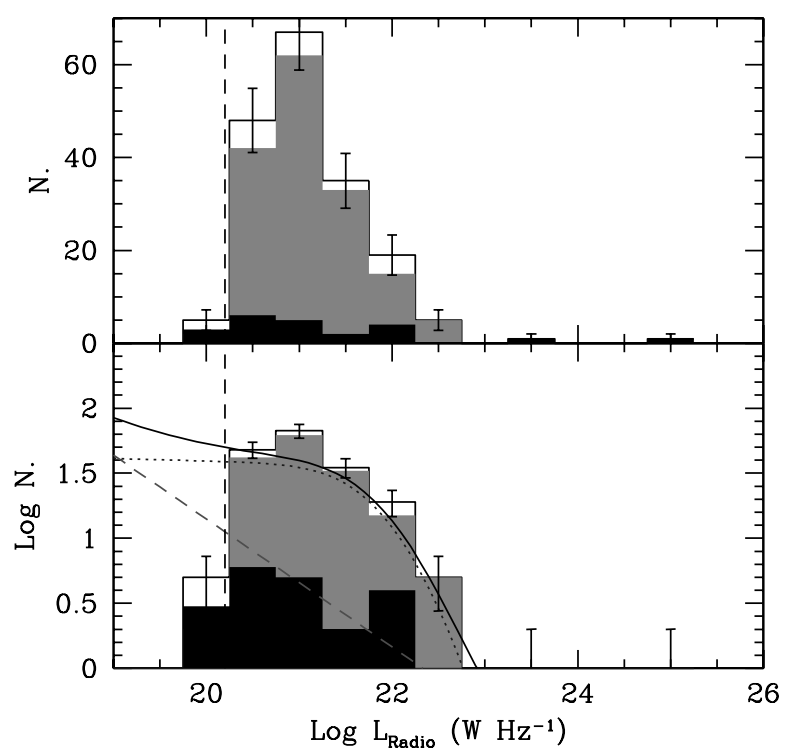

FIG. 7.-Radio continuum (1415 MHz) luminosity distribution in linear (upper panel) and logarithmic (lower panel) scales for the detected HRS galaxies (empty histogram). The black and gray histograms are for early-type (E-S0-SOa) and late-type (Sa-Sd-Im-BCD) galaxies, respectively, compared to the 2dF/NVSS luminosity function of Mauch \& Sadler (2007, solid line). This radio luminosity function includes both the contribution of AGN (dashed line) and quiescent (dotted line) galaxies. Vertical dashed line indicates the typical detection limit of NVSS (2.5 mJy) at the distance of $20 \mathrm{Mpc}$. See the electronic edition of the PASP for a color version of this figure.

universe LIRG and ULIRG are excluded. The HRS matches also the distribution of a radio-continuum selected sample, although the statistic for the brightest radio galaxies is poor. However, Figure 4 shows that when viewed from the perspective of gas mass, the HRS is not a fair sample of the local universe. The presence of HI-deficient objects makes it ideal for studying the effects of the cluster environment on the dust properties of nearby galaxies.

We would like to thank the Herschel Project Scientist, G. Pilbratt; the SPIRE team; and all the people involved in the construction and the launch of Herschel. This research has made use of the NASA/IPAC Extragalactic Database (NED) which is operated by the Jet Propulsion Laboratory, California Institute of Technology, under contract with the National Aeronautics and Space Administration; and of the GOLD Mine database. A. B. wishes to thank S. Boissier for his help in the construction of the HRS database. We are grateful to the anonymous referee forinvaluable comments and suggestions which helped to improve the quality of the manuscript.

\section{REFERENCES}

Adelman-McCarthy, J., et al. 2008, ApJS, 175, 297

Alton, P., Trewhella, M., \& Davies, J., et al. 1998, A\&A, 335, 807
Alton, P., Trewhella, M., Davies, J., \& Bianchi, S. 1999, A\&A, 343, 51

Beker, R., White, R., \& Helfand, D. 1995, ApJ, 450, 559 
Bendo, G., Joseph, R., \& Wells, M., et al. 2003, AJ, 125, 2361

Binggeli, B., Sandage, A., \& Tammann, G. 1985, AJ, 90, 1681

Böhringer, H., Briel, U. G., Schwarz, R. A., Voges, W., Hartner, G., \& Trumper, J. 1994, Nature, 368, 828

Boselli, A., \& Gavazzi, G. 2002, A\&A, 386, 124

- 2006, PASP, 118, 517

. 2009, A\&A, 508, 201

Boselli, A., Casoli, F., \& Lequeux, J. 1995, A\&AS, 110, 521

Boselli, A., Tuffs, R., Gavazzi, G., Hippelein, H., \& Pierini, D. 1997, A\&A, 121, 507

Boselli, A., Gavazzi, G., Franzetti, P., Pierini, D., \& Scodeggio, M. 2000, A\&AS, 142, 73

Boselli, A., Gavazzi, G., Donas, J., \& Scodeggio, M. 2001, AJ, 121, 753

Boselli, A., Lequeux, J., \& Gavazzi, G. 2002a, A\&A, 384, 33

Boselli, A., Iglesias-Páramo, J., Vilchez, J. M., \& Gavazzi, G. 2002b, A\&A, 386, 134

Boselli, A., Gavazzi, G., Lequeux, J., \& Pierini, D. 2002c, A\&A, 385,454

Boselli, A., Gavazzi, G., \& Sanvito, G. 2003a, A\&A, 402, 37

Boselli, A., Sauvage, M., Lequeux, J., Donati, A., \& Gavazzi, G. 2003b, A\&A, 406, 867

Boselli, A., Lequeux, J., \& Gavazzi, G. 2004, A\&A, 428, 409

Boselli, A., Cortese, L., \& Deharveng, J. M., et al. 2005a, ApJ, 629, L29

Bower, R. G., Lucey, J. R., \& Ellis, R. S. 1992, MNRAS, 254, 601

Bregman, J., Snider, B., Grego, L., \& Cox, C. 1998, ApJ, 499, 670

Bressan, A., Granato, G. L., \& Silva, L. 1998, A\&A, 332, 135

Bressan, A., Panuzzo, P., \& Buson, L., et al. 2006, ApJ, 639, L55

Broelis, A. H., \& Van Woerden, H. 1994, A\&AS, 107, 129

Buat, V., \& Xu, K. 1996, A\&A, 306, 61

Buat, V., Boselli, A., Gavazzi, G., \& Bonfanti, C. 2002, A\&A, 383, 801

Calzetti, D. 2001, PASP, 113, 1449

Calzetti, D., Kennicutt, R., \& Bianchi, L., et al. 2005, ApJ, 633, 871

Calzetti, D., Kennicutt, R., \& Engelbracht, C., et al. 2007, ApJ, 666,870

Catinella, B., Giovanelli, R., \& Haynes, M. 2006, ApJ, 640, 751

Cayatte, V., Kotanyi, C., Balkowski, C., \& van Gorkom, J. 1994, AJ, 107,1003

Cayatte, V., van Gorkom, J., Balkowski, C., \& Kotanyi, C. 1990, AJ, 100,604

Chanial, P., Flores, H., Guiderdoni, B., Elbaz, D., Hammer, F., \& Vigroux, L. 2007, A\&A, 462, 81

Chung, A., van Gorkom, J., Kenney, J., Crowl, H., \& Vollmer, B. 2009, AJ, 138, 1741

Cole, S., Norberg, P., \& Baugh, C., et al. 2001, MNRAS, 326, 255

Condon, J. J., Cotton, W. D., \& Broderick, J. J. 2002, AJ, 124, 675

Condon, J. J., Cotton, W. D., Greisen, E. W., Yin, Q. F., Perley, R. A., Taylor, G. B., \& Broderick, J. J. 1998, AJ, 115, 1693

Cortese, L., Boselli, A., \& Buat, V., et al. 2006, ApJ, 637, 242

Cortese, L., Boselli, A., Franzetti, P., Decarli, R., Gavazzi, G., Boissier, S., \& Buat, V. 2008a, MNRAS, 386, 1157

Cortese, L., Minchin, R., \& Auld, R., et al. 2008b, MNRAS, 383, 1519

Cortese, L., \& Hughes, T. 2009, MNRAS, 400, 1225

Cowie, L., Songaila, A., Hu, E., \& Cohen, J. 1996, AJ, 112, 839

Cuillandre, J. C., Lequeux, J., Allen, R., Mellier, Y., \& Bertin, E. 2001, ApJ, 554, 190

Dale, D., Bendo, G., \& Engelbracht, C., et al. 2005, ApJ, 633, 857
Dale, D., Gil de Paz, A., \& Gordon, K., et al. 2007, ApJ, 655, 863

Davies, J., Alton, P., Bianchi, S., \& Trewhella, M. 1998, MNRAS, 300, 1006

Davies, J., Alton, P., Trewhella, M., Evans, R., \& Bianchi, S. 1999, MNRAS, 304, 495

Désert, F.-X., Boulanger, F., \& Puget, J.-L. 1990, A\&A, 237, 215

Devereux, N., \& Young, J. 1990, ApJ, 359, 42

Disney, M., Davies, J., \& Phillipps, S. 1989, MNRAS, 239, 939

Djorgovski, S., \& Davis, M. 1987, ApJ, 313, 59

Draine, B. 1978, ApJS, 36, 596

Draine, B., \& Li, A. 2007, ApJ, 657, 810

Draine, B., Dale, D., \& Bendo, G., et al. 2007, ApJ, 663, 866

Dressler, A., Lynden-Bell, D., Burstein, D., Davies, R., Faber, S., Terlevich, R., \& Wegner, G. 1987, ApJ, 313, 42

Duley, W., \& Williams, D. 1986, MNRAS, 223, 177

Dunne, L., \& Eales, S. 2001, MNRAS, 327, 697

Dunne, L., Eales, S., Edmunds, M., Ivison, R., Alexander, P., \& Clements, D. 2000, MNRAS, 315, 115

Dwek, E. 1986, ApJ, 302, 363

Dwek, E., Arendt, R., \& Fixsen, D., et al. 1997, ApJ, 475, 565

Eales, S., Wynn-Williams, C., \& Duncan, W. 1989, ApJ, 339, 859

Engelbracht, C., Kundurthy, P., \& Gordon, K., et al. 2006, ApJ, 642, L127

Fabbiano, G., Kim, D., \& Trinchieri, G. 1992, ApJS, 80, 531

Ferrarese, L., Côté, P., \& Jordán, A., et al. 2006, ApJS, 164, 334

Ferrari, F., Pastoriza, M. G., Macchetto, F., \& Caon, N. 1999, A\&AS, 136, 269

Galliano, F., Madden, S., Jones, A., Wilson, C., Bernard, J-P., \& Le Peintre, F. 2003, A\&A, 407, 159

Galliano, F., Madden, S., Jones, A., Wilson, C., \& Bernard, J-P. 2005, A\&A, 434, 867

Galliano, F., Dwek, E., \& Chanial, P. 2008, ApJ, 672, 214

Gallo, E., Treu, T., Jacob, J., Woo, J. H., Marshall, P., \& Antonucci, R. 2008, ApJ, 680, 154

Garcia-Barreto, J., Dowens, D., \& Huchtmeier, W. 1994, A\&A, 288,705

Gavazzi, G., Pierini, D., \& Boselli, A. 1996, A\&A, 312, 397

Gavazzi, G., Catinella, B., Carrasco, L., Boselli, A., \& Contursi, A. 1998, AJ, 115, 1745

Gavazzi, G., Boselli, A., Scodeggio, M., Pierini, D., \& Belsole, E. 1999a, MNRAS, 304, 595

Gavazzi, G., Carrasco, L., \& Galli, R. 1999b, A\&AS, 136, 227

Gavazzi, G., \& Boselli, A. 1999c, A\&A, 343, 86

1999d, A\&A, 343, 93

Gavazzi, G., Franzetti, P., Scodeggio, M., Boselli, A., \& Pierini, D. 2000a, A\&A, 361, 863

Gavazzi, G., Franzetti, P., Scodeggio, M., Boselli, A., Pierini, D., Baffa, C., Lisi, F., \& Hunt, L. 2000b, A\&AS, 142, 65

Gavazzi, G., Boselli, A., Pedotti, P., Gallazzi, A., \& Carrasco, L. 2002a, A\&A, 396, 449

Gavazzi, G., Bonfanti, C., Sanvito, G., Boselli, A., \& Scodeggio, M. 2002b, ApJ, 576, 135

Gavazzi, G., Boselli, A., Donati, A., Franzetti, P., \& Scodeggio, M. 2003, A\&A, 400, 451

Gavazzi, G., Zaccardo, A., Sanvito, G., Boselli, A., \& Bonfanti, C. 2004, A\&A, 417, 499

Gavazzi, G., Boselli, A., van Driel, W., \& O’Neil, K. 2005, A\&A, 429,439 
Gavazzi, G., Boselli, A., Cortese, L., Arosio, I., Gallazzi, A., \& Pedotti, P. 2006, A\&A, 446, 839

Gavazzi, G., Giovanelli, R., \& Haynes, M., et al. 2008, A\&A, 482, 43 Gerin, M., \& Casoli, F. 1994, A\&A, 290, 49

Giovanelli, R., Haynes, M., \& Kent, B., et al. 2005, AJ, 130, 25, 98 Gil de Paz, A., Boissier, S., \& Madore, B., et al. 2007, ApJS, 173, 185

Gordon, K., Perez-Gonzalez, P., \& Misselt, K., et al. 2004, ApJS, 154,215

Gordon, K., Rieke, G. H., \& Engelbracht, C. W., et al. 2005, PASP, 117,831

Goudfrooij, P., Hansen, L., Jorgensen, H. E., \& Norgaard-Nielsen, H. U. 1994, A\&AS, 105, 341

Griffin, M., Abergel, A., Ade, P., André, P., Baluteau, J-P., Bock, J., Franceschini, A., \& Gear, W., et al. 2007, Adv. Space Res., 40 (5), 612

2006, Proc. SPIE, 6265, 7

Guélin, M., Zylka, R., \& Mezger, P. G., et al. 1993, A\&A, 279, L37

Guélin, M., Zylka, R., Mezger, P. G., Haslam, C. G. T., \& Kreysa, E. 1995, A\&A, 298, L29

James, A., Dunne, L., Eales, S., \& Edmunds, M. 2002, MNRAS, 335,753

Jansen, R., Fabricant, D., Franx, M., \& Caldwell, N. 2000, ApJS, 126,331

Jarrett, T., Chester, T., Cutri, R., Schneider, S., \& Huchra, J. 2003, AJ, 125,525

Haynes, M., \& Giovanelli, R. 1984, AJ, 89, 758

Heavens, A., Panter, B., Jimenez, R., \& Dunlop, J. 2004, Nature, 428,625

Hirashita, H., Buat, V., \& Inoue, A. 2003, A\&A, 410, 83

Hollenbach, D., \& Salpeter, E. 1971, ApJ, 163, 155

Hollenbach, D., \& Tielens, A. 1997, ARA\&A, 35, 179

Holmberg, E. 1958, Lund Medd. Astron. Obs. Ser. II, 136, 1

Hughes, T., Cortese, L. 2009, MNRAS, 396, L41

Kaneda, H., Onaka, T., Kitayama, T., Okada, Y., \& Sakon, I. 2007, PASJ, 59, 107

Karachentsev, I., Lebedev, V., \& Shcherbanovskij, A. 1972, Publ. Special Astrophys. Obs. USSR 7

Kauffmann, G., Heckman, T., \& White, S., et al. 2003, MNRAS, 341,54

Kenney, J., \& Young, J. 1988, ApJS, 66, 261

Kennicutt, R. 1992a, ApJ, 388, 310

1992b, ApJS, 79, 255

1998, ARA\&A, 36, 189

Kennicutt, R., Armus, L., \& Bendo, G., et al. 2003, PASP, 115, 928

Kennicutt, R., Calzetti, D., \& Walter, F., et al. 2007, ApJ, 671, 333

Kochanek, C., Pahre, M., Falco, E., Huchra, J., Mader, J., Jarrett, T., Chester, T., Cutri, R., \& Schneider, S. 2001, ApJ, 560, 566

Leech, K., Völk, H., \& Heinrichsen, I., et al. 1999, MNRAS, 310, 317

Leeuw, L., Hawarden, T., Matthews, H., Robson, I., \& Eckart, A. 2002, ApJ, 565, L131

Leeuw, L., Davidson, J., Dowell, K., \& Matthews, H. 2008, ApJ, 677, L249

Malhotra, S., Kaufman, M., \& Hollenbach, D., et al. 2001, ApJ, 561, 766

Mauch, T., \& Sadler, E. 2007, MNRAS, 375, 931

Meiksin, A. 2009, Rev. Mod. Phys., 81, 1405

Montier, L., \& Giard, M. 2005, A\&A, 439, 35

Morton, R., \& Haynes, M. 1994, ARA\&A, 32, 115
Moustakas, J., \& Kennicutt, R. 2006, ApJS, 164, 81

Neininger, N., Guélin, M., Garcia-Burillo, S., Zylka, R., \& Wielebinski, R. 1996, A\&A, 310, 725

Niklas, S., Klein, U., \& Wielebinski, R. 1995, A\&A, 293, 56

Nilson, P. 1973, The Uppsala General Catalogue of Galaxies (Uppsala: Uppsala Univ. Press)

Nolthenius, R. 1993, ApJS, 85, 1

Oppenheimer, B., \& Davé, R. 2008, MNRAS, 387, 577

Panuzzo, P., Vega, O., \& Bressan, A., et al. 2007, ApJ, 656, 206

Perez-Gonzalez, P., Kennicutt, R., \& Gordon, K., et al. 2006, ApJ, 648, 987

Prescott, M., Kennicutt, R., \& Bendo, G., et al. 2007, ApJ, 668, 182

Rayan-Weber, E., Pettini, M., \& Madau, P. 2006, MNRAS, 371, L78

Roussel, H., Vigroux, L., \& Bosma, A., et al. 2001b, A\&A, 369, 473

Sarazin, C. 1986, Rev. Mod. Phys., 58, 1

Schlegel, F., Finkbeiner, D., \& Davis, M. 1998, ApJ, 500, 525

Scodeggio, M., Gavazzi, G., Franzetti, P., Boselli, A., Zibetti, S., \& Pierini, D. 2002, A\&A, 384, 812

Shapley, A., Fabbiano, G., \& Eskridge, P. 2001, ApJS, 137, 139

Skrutskie, M., Cutri, R., \& Stiening, R., et al. 2006, AJ, 131, 1163

Soifer, B., Neugebauer, G., \& Houck, J. 1987, ARA\&A, 25, 187

Springob, C., Haynes, M., Giovanelli, R., \& Kent, B. 2005, ApJS, 160,149

Stickel, M., Klaas, U., Lemke, D., \& Mattila, D. 2002, A\&A, 383, 367

Takeuchi, T., Yoshikawa, K., \& Ishii, T. 2003, ApJ, 587, L89

Temi, P., Brighenti, F., \& Mathews, W. 2007, ApJ, 660, 1215

Tielens, A., McKee, C., Seab, C., \& Hollenbach, D. 1994, ApJ, 431, 321

Thilker, D., Boissier, S., \& Bianchi, L., et al. 2007, ApJS, 173, 572

Thomas, H., Dunne, L., Clemens, M., Alexander, P., Eales, S., Green, D., \& James, A. 2002, MNRAS, 331, 853

Tremonti, C., Heckman, T., \& Kauffmann, G., et al. 2004, ApJ, 613, 898

Tuffs, R., Popescu, C., \& Pierini, D., et al. 2002, ApJS, 139, 37

Tully, B., \& Fisher, J. 1977, A\&A, 54, 661

Tully, B., Mould, J., \& Aaronson, M. 1982, ApJ, 257, 527

Tully, B. 1988, Nearby Galaxy Catalog (Cambridge: Cambridge Univ. Press)

van der Hulst, J. 2002, in ASP Conf. Proc. 276, Seeing Through the Dust: The Detection of HI and the Exploration of the ISM in Galaxies, ed. A. R., Taylor, T. L., Landecker, \& A. G., Willis (San Francisco: ASP) 84

Van Dokkum, P. G., \& Franx, M. 1995, ApJ, 110, 2027

Verheijen, M., Sancisi, R. 2001, A\&A, 370, 765

Visvanathan, N., Sandage, A. 1977, ApJ, 216, 214

Vlahakis, C., Dunne, L., Eales, S. 2005, MNRAS, 364, 1253

Voges, W., Aschenbach, B., \& Boller, T. 1999, A\&A, 349, 389

Vollmer, B., Thierbach, M., Wielebinski, R. 2004, A\&A, 418, 1

Ward-Thompson, D., André, P., Kirk, J. 2002, MNRAS, 329, 257

Waskett, T., Sibthorpe, B., Griffin, J., Chanial, P. F. 2007, MNRAS, 381,1583

Whittet, D. 1992, in Dust in the galactic environment (Graduate Series in Astronomy; Bristol: IOP Publishing)

Witt, A., Gordon, K. 2000, ApJ, 528, 799

Xilouris, E., Madden, S., Galliano, F., Virgoux, L., Sauvage, M. 2004, A\&A, 416, 41

Young, J., Xie, S., Tacconi, L., et al. 1995, ApJS, 98, 219 
Young, L., Bendo, G., Lucero, D. 2008 in The Evolving ISM in the Milky Way and Nearby Galaxies, 4th Spitzer Science Center Conf. (Pasadena, December 2007

Zaritsky, D., Kennicutt, R., Huchra, J. 1994, ApJ, 420, 87

Zubko, V., Dwek, E., Arendt, R. 2004, ApJS, 152, 211
Zwaan, M., Meyer, M., Staveley-Smith, L., \& Webster, R. 2005, MNRAS, 359, L30

Zwicky, F., Herzog, E., Karpowicz, M., Kowal, C., \& Wild, P. 1961 1968, Catalogue of Galaxies and of Cluster of Galaxies (CGCG; Pasadena: Caltech) 